\title{
THE KAKEYA PROBLEM FOR CIRCULAR ARCS
}

\author{
K. Héra and M. Laczkovich
}

August 12, 2016

\begin{abstract}
We prove that if a circular arc has angle short enough, then it can be continuously moved to any prescribed position within a set of arbitrarily small area.
\end{abstract}

\section{Introduction and main results}

It is well-known that a line segment can be continuously moved in a plane set of arbitrarily small area such that it returns to its starting position, with its direction reversed. This fact was first proved by Besicovitch as a solution to the classical Kakeya problem [1. Besicovitch's construction used the socalled 'Pál joins' in order to shift the line segment to an arbitrary parallel position using arbitrarily small area. Using these Pál joins, one can easily deduce from Besicovitch's theorem that every line segment can be moved to arrive at any prescribed position within a set of arbitrarily small area.

In the paper 'Three Kakeya problems' F. Cunningham asked whether or not a circular arc has a similar property [3, p. 591]. Our aim is to show that the answer is affirmative, at least for circular arcs of angle short enough.

\footnotetext{
${ }^{1}$ Keywords: Kakeya problem for circular arcs

${ }^{2} \mathrm{MSC} 2010: 28 \mathrm{~A} 75$

${ }^{3}$ The research of the second author was partially supported by the Hungarian National Research, Development and Innovation Office, Grant No. NKFIH 104178
} 
Theorem 1.1. Let $\sigma$ be a circular arc in the plane of radius 1 and of arc length less than 1.32. Then for every $\varepsilon>0$, we can move $\sigma$ continuously to any given position such that the area of the points touched by the moving arc is less than $\varepsilon$.

In [4] we introduced the following terminology. We say that the set $A \subset$ $\mathbb{R}^{2}$ has property (K), if there exist two different positions of $A$ such that $A$ can be continuously moved from the first position to the second within a set of arbitrarily small area. It is obvious that all line segments and circles have property $(\mathrm{K})$.

The set $A$ is said to have property $\left(\mathrm{K}^{\mathrm{s}}\right)$ if it can be moved to arrive at any prescribed position within a set of arbitrarily small area. As we saw above, line segments have property $\left(\mathrm{K}^{\mathrm{s}}\right)$. According to Theorem 1.1, those circular arcs which have angle short enough also have property $\left(\mathrm{K}^{\mathrm{s}}\right)$. On the other hand, (full) circles do not have property $\left(\mathrm{K}^{\mathrm{s}}\right)$ since every continuous movement placing the circle far enough must touch every point inside the circle. We do not know whether or not all circular arcs shorter than the full circle have property $\left(\mathrm{K}^{\mathrm{s}}\right)$, as our construction does not seem to work in the case where the angle of the arc is longer than a certain bound. We remark that apart from line segments and circular arcs no continuum can have property $\left(\mathrm{K}^{\mathrm{s}}\right)$ (see [4, Theorem 1.2]).

We can easily reduce Theorem 1.1 to the case when the final position is obtained from $\sigma$ by rotating it about one of its endpoints.

Theorem 1.2. Let $\sigma_{0}$ be a circular arc of radius 1 and of arc length less than 1.32 , and let $\sigma_{1}$ be obtained from $\sigma_{0}$ by rotating it about one of its endpoints. Then, for every $\varepsilon>0$, we can move $\sigma_{0}$ continuously to $\sigma_{1}$ such that the area of the points touched by the moving arc is less than $\varepsilon$.

Assuming Theorem 1.2, we can prove Theorem 1.1 as follows. Let $\sigma_{0}$ and $\sigma_{1}$ be congruent circular arcs of radius 1 and of arc length less than 1.32. Let $O_{0}$ and $O_{1}$ be the centres of the circles of $\sigma_{0}$ and $\sigma_{1}$, respectively. Let $O_{0}=P_{0}, P_{1}, \ldots, P_{n}=O_{1}$ be points such that $0<\left|P_{i-1} P_{i}\right|<2$ for every $i=1, \ldots, n$, where $|A B|$ denotes the distance between the points $A$ and $B$. Let $K_{i}$ denote the circle with centre $P_{i}$ and radius $1(i=0, \ldots, n)$. Then, for every $i=1, \ldots, n$, the circles $K_{i-1}$ and $K_{i}$ have two common points; let $M_{i}$ be one of them. 
Let $\varepsilon>0$ be given. First we rotate the arc $\sigma$ with initial position $\sigma_{0}$ about the point $O_{0}=P_{0}$ until one of its endpoints becomes $M_{1}$. Then, using Theorem 1.2 , we apply a suitable continuous motion touching an area less than $\varepsilon / n$ which places $\sigma$ into the circle $K_{1}$. Then we rotate $\sigma$ about the point $P_{1}$ until one of its endpoints becomes $M_{2}$. Using Theorem 1.2, we apply a suitable continuous motion touching an area less than $\varepsilon / n$ which places $\sigma$ into the circle $K_{2}$. Iterating this process $n$ times, $\sigma$ will be moved to a subarc of $K_{n}$. Then, using a suitable rotation about the point $P_{n}=O_{1}$ we reach the final position $\sigma_{1}$. It is clear that the area of the set of points touched by this motion is less than $\varepsilon$, which proves Theorem 1.1 .

We shall prove Theorem 1.2 in the next 5 sections. After stating some preliminary lemmas in the next section, we describe the basic construction, an adaptation of Cunningham's sprouting process (see [2, pp. 118-120]), in Section 3. We estimate the area touched by the moving arc in Section 4, and then, using this estimate, we prove Theorem 1.2 in Section 5 . The proofs of the lemmas stated and used during the proof are given in Section 6 .

\section{Preliminary lemmas}

Lemma 2.1. Let $K$ be a circle of radius 1 and centre $O$, and let $P$ be a point such that $\operatorname{dist}(P, K)=d<1 / 2$. Let $Q \in K$ be a point such that the orientation of the triangle $\Delta_{P O Q}$ is positive (counter-clockwise).

(i) If $P$ is in the interior of $K$ and $|P Q| \geq 2 \sqrt{d}$, then there is an angle $0<\alpha<\pi / 2$ such that $\sin \alpha<2 \sqrt{d}$, and rotating $K$ about the point $Q$ in the positive direction by angle $\alpha$, the circle $K^{\prime}$ obtained contains $P$.

(ii) If $P$ is in the exterior of $K$ and $3 \sqrt{d} \leq|P Q| \leq 2-d$, then there is an angle $0<\alpha<\pi / 2$ such that $\sin \alpha<2 \sqrt{d}$, and rotating $K$ about the point $Q$ in the negative direction by angle $\alpha$, the circle $K^{\prime}$ obtained contains $P$.

Moreover, the distance between $O$ and the centre of $K^{\prime}$ is less than $4 \sqrt{d}$.

Figures 5 and 6 on pp. 20 and 21 illustrate the two cases of Lemma 2.1. 
Lemma 2.2. Let $K$ be a circle of radius 1 and centre $O$. Let $A, B \in K$ be such that the orientation of the triangle $\Delta_{O A B}$ is positive, and $\eta=B O A \angle<$ $1 / 5$. Let $K_{A}$ and $K_{B}$ be the circles obtained by rotating $K$ about the points $A$ and $B$ in the positive direction by the angles $\alpha$ and $\beta$, respectively, where $0<\alpha<3 \beta / 4$ and $\beta<\eta$. Then one of the intersection points of $K_{A}$ and $K_{B}$ is inside $K$, and its distance from $A$ is less than $20 \eta$.

See Figure 7 on page 22 .

Lemma 2.3. Let $K$ be a circle of radius 1 and centre $O$. Let $A, B \in K$ be such that the orientation of the triangle $\Delta_{O A B}$ is positive, and $\eta=B O A \angle<$ 1/10. Let $K_{A}$ and $K_{B}$ be the circles obtained by rotating $K$ about the points $A$ and $B$ in the negative direction by the angles $\alpha$ and $\beta$, respectively, where $0<\alpha<3 \beta / 4$ and $\beta<\eta$. Then one of the intersection points of $K_{A}$ and $K_{B}$ is outside $K$, and its distance from $A$ is less than $50 \eta$.

See Figure 8 on page 25.

Lemma 2.4. Let $K$ be a circle of radius $r$, and let $\overparen{A B}$ be a subarc of $K$ of length $<r \pi$. Rotating the arc $\overparen{A B}$ about the point $A$ by an angle $\alpha$ we obtain the arc $\overparen{A C}$. Then the area of the domain $H$ bounded by the arcs $\widehat{A B}, \widehat{A C}$ and $\overparen{B C}$ is $|A B|^{2} \cdot \alpha / 2$.

In the next two lemmas we shall use the following notation. Let $h, \varepsilon$ be positive numbers satisfying $\varepsilon<10^{-6}$ and $h \leq \varepsilon / 10^{3}$. Let $K_{0}$ and $K_{1}$ be the circles of radius 1 and centres $(-\sin (h / 2), 0)$ and $(\sin (h / 2), 0)$, respectively. The point with coordinates $(0, \cos (h / 2))$ will be denoted by $M$. Thus $M \in$ $K_{0} \cap K_{1}$.

Let $\bar{K}_{i}$ denote the disc bounded by $K_{i}(i=0,1)$. The closures of the sets $\bar{K}_{0} \backslash \bar{K}_{1}$ and $\bar{K}_{1} \backslash \bar{K}_{0}$ are denoted by $L_{0}$ and $L_{1}$.

The circle of radius $\varepsilon$ and centre $M$ intersects the lune $L_{0}$ in the arc $\widehat{P_{0} P_{1}}$, where $P_{0} \in K_{0}$ and $P_{1} \in K_{1}$.

Lemma 2.5. If a line intersects the arc $\widehat{P_{0} P_{1}}$ at two points, or touches the arc $\widehat{P_{0} P_{1}}$, then the angle between the line and the $y$ axis is less than $h+\varepsilon$.

Lemma 2.6. Let $Q^{\prime}$ and $Q^{\prime \prime}$ be points of the arc $\widehat{P_{0} P_{1}}$ such that the $y$ coordinate of $Q^{\prime}$ is greater than the $y$ coordinate of $Q^{\prime \prime}$. Let $C \in L_{1}$ such that 
$|C M| \leq 2-5 \varepsilon$, and let $K^{\prime}$ and $K^{\prime \prime}$ be circles with the following properties. The circle $K^{\prime}$ has radius 1 and centre $O^{\prime}$, contains the points $Q^{\prime}$ and $C$, the circle $K^{\prime \prime}$ has radius 1 and centre $O^{\prime \prime}$, and contains the points $Q^{\prime \prime}$ and $C$, and $\left|O O^{\prime}\right|,\left|O O^{\prime \prime}\right|<\varepsilon$.

Let $\rho$ denote the rotation about the point $C$ mapping $K^{\prime \prime}$ onto $K^{\prime}$ and let the angle of the rotation $\rho$ be $\alpha$. Then

(i) $\alpha<\varepsilon$,

(ii) the angle between the $x$ axis and the line going through the points $Q^{\prime}$ and $\rho\left(Q^{\prime \prime}\right)$ is less than $6 \varepsilon$.

\section{The sprouting process}

First we fix three parameters: the positive numbers $h, \varepsilon$ satisfying $\varepsilon<10^{-6}$ and $h<\varepsilon / 10^{3}$, and the positive integer $n$.

Let $K_{0}$ and $K_{1}$ be the circles of radius 1 and centres $O_{0}=(-\sin (h / 2), 0)$ and $O_{1}=(\sin (h / 2), 0)$, respectively. The intersection $K_{0} \cap K_{1}$ consists of the points $M, N$ lying on the $y$ axis, where the $y$ coordinate of $M$ is positive, and the $y$ coordinate of $N$ is negative. Then the triangle with vertices $O_{0}, M, O_{1}$ is isosceles, and $O_{0} M O_{1} \angle=h$. This means that rotating $K_{0}$ about the point $M$ by angle $h$ in the positive direction we obtain $K_{1}$.

We put $R=1.227<2-5 \varepsilon$. Since $|M N|=2 \cos (h / 2)>2-h>2-\varepsilon$, we have $R<|M N|$.

Let $\bar{K}_{i}$ denote the disc bounded by $K_{i}(i=0,1)$. The closures of the sets $\bar{K}_{0} \backslash \bar{K}_{1}$ and $\bar{K}_{1} \backslash \bar{K}_{0}$ are denoted by $L_{0}$ and $L_{1}$.

The circle of radius $\varepsilon$ and centre $M$ intersects the lune $L_{0}$ in the arc $\widehat{P_{0} P_{1}}$, where $P_{0} \in K_{0}$ and $P_{1} \in K_{1}$. The length of $\widehat{P_{0} P_{1}}$ equals $h \varepsilon$, since $P_{0} M P_{1} \angle=h$.

For every $x \in[0,1], P_{x}$ will denote the point of the arc $\overparen{P_{0} P_{1}}$ such that the length of the $\operatorname{arc} \widehat{P_{0} P_{x}}$ equals $x$ times the length of the arc $\overparen{P_{0} P_{1}}$. Thus $P_{1 / 2}$ is the middle point of the $\operatorname{arc} \widehat{P_{0} P_{1}}$.

We put $r_{i}=i R / n(i=1, \ldots, n)$. The circle of radius $r_{i}$ and centre $M$ intersects the lune $L_{1}$ in the arc $\widehat{A_{i}^{0} A_{i}^{1}}$, where $A_{i}^{0} \in K_{0}$ and $A_{i}^{1} \in K_{1}$ 


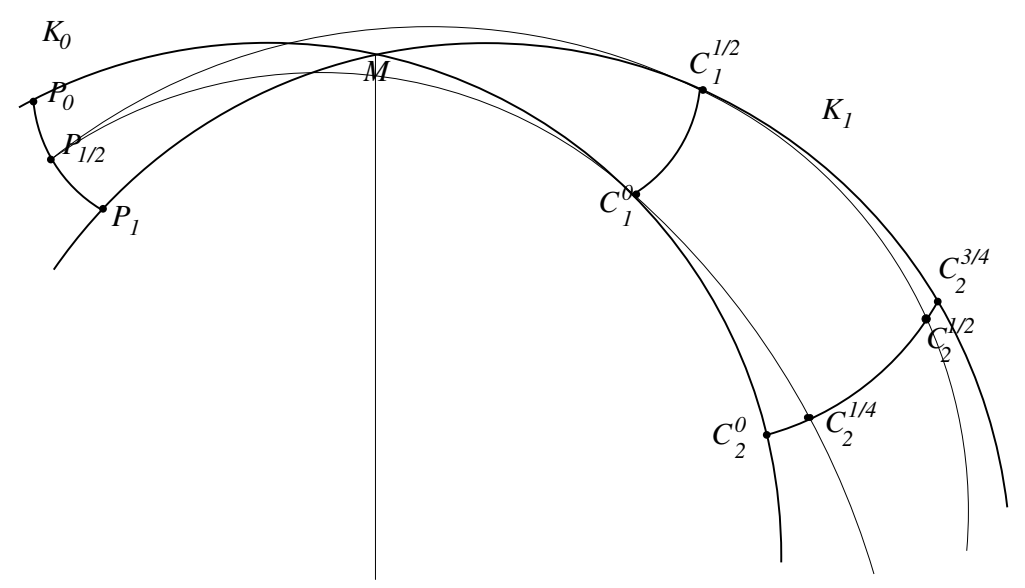

Figure 1: Sprouting

$(i=1, \ldots, n)$. We define $A_{0}^{0}=A_{0}^{1}=M$.

We denote by $H$ the horn shaped domain contained in $L_{1}$, bounded by the circles $K_{0}, K_{1}$ and the circle of radius $R$ and centre $M$. Then the diameter of $H$ is the distance $\left|A_{n}^{0} M\right|=\left|A_{n}^{1} M\right|=R$.

The 'sprouting process' is a construction of a system of circles of radius 1 inductively. We start with the circles $K_{0}$ and $K_{1}$. We put $K_{0}^{0}=K_{0}$ and $K_{1}^{1}=K_{1}$. We rotate $K_{0}^{0}$ about the point $A_{1}^{0}$ such that the resulting circle, denoted by $K_{1}^{1 / 2}$, contains $P_{1 / 2}$. Similarly, we rotate $K_{1}^{1}$ about the point $A_{1}^{1}$ such that the resulting circle, denoted by $K_{0}^{1 / 2}$, contains $P_{1 / 2}$.

In this way we have defined the circles $K_{0}^{0}, K_{1}^{1 / 2}, K_{0}^{1 / 2}, K_{1}^{1}$ such that $P_{0} \in$ $K_{0}^{0}, P_{1 / 2} \in K_{0}^{1 / 2} \cap K_{1}^{1 / 2}$ and $P_{1} \in K_{1}^{1}$. Using these circles we can transform $K_{0}$ to $K_{1}$ by rotating $K_{0}=K_{0}^{0}$ about $A_{1}^{0}$ to obtain $K_{1}^{1 / 2}$, then rotating the latter about the point $P_{1 / 2}$ to obtain $K_{0}^{1 / 2}$, and then rotating the latter about $A_{1}^{1}$ to obtain $K_{1}^{1}=K_{1}$.

We continue the process as follows. Let $K_{0}^{0}, K_{1}^{1 / 2}, K_{0}^{1 / 2}, K_{1}^{1}$ intersect the $\operatorname{arc} \widehat{A_{2}^{0} A_{2}^{1}}$ at the points $C_{2}^{0}, C_{2}^{1 / 4}, C_{2}^{1 / 2}, C_{2}^{3 / 4}$, respectively. Thus $C_{2}^{0}=A_{2}^{0}$ and $C_{2}^{3 / 4}=A_{2}^{1}$ (see Figure 1 ).

Rotating $K_{0}^{0}$ about $C_{2}^{0}$ we obtain $K_{1}^{1 / 4}$ containing $P_{1 / 4}$, rotating $K_{1}^{1 / 2}$ about $C_{2}^{1 / 4}$ we obtain $K_{0}^{1 / 4}$ containing $P_{1 / 4}$, rotating $K_{0}^{1 / 2}$ about $C_{2}^{1 / 2}$ we 
obtain $K_{1}^{3 / 4}$ containing $P_{3 / 4}$, and rotating $K_{1}^{1}$ about $C_{2}^{3 / 4}$ we obtain $K_{0}^{3 / 4}$ containing $P_{3 / 4}$. Then, using the circles $K_{0}^{0}, \ldots, K_{0}^{3 / 4}$ and $K_{1}^{1 / 4}, \ldots, K_{1}^{1}$ we can transform $K_{0}$ to $K_{1}$ by using rotations about the points $C_{2}^{0}, \ldots, C_{2}^{3 / 4}$ and $P_{1 / 4}, P_{1 / 2}, P_{3 / 4}$ alternately.

Now we describe the general construction in detail. We denote by $D_{i}$ the set $\left\{k / 2^{i}: 0 \leq k \leq 2^{i}\right\}$ for every $i=0,1, \ldots$ We define the point $C_{i}^{x}$ and the circle $K_{0}^{x}$ for every $0 \leq i \leq n, x \in D_{i}, x<1$, and the circle $K_{1}^{x}$ for every $0 \leq i \leq n, x \in D_{i}, x>0$ satisfying the following conditions. For every $0 \leq i \leq n$ we have

$$
\begin{gathered}
C_{i}^{x} \in \widehat{A_{i}^{0} A_{i}^{1}} \quad\left(x \in D_{i}, x<1\right) ; \\
K_{0}^{x} \text { contains the points } P_{x} \text { and } C_{i}^{x} \quad\left(x \in D_{i}, x<1\right) ; \\
K_{1}^{x} \text { contains the points } P_{x} \text { and } C_{i}^{x-2^{-i}} \quad\left(x \in D_{i}, x>0\right) ;
\end{gathered}
$$

the centres of the circles $K_{0}^{x}$ and $K_{1}^{x+2^{-i}}$ are closer to the origin than

$$
h+3 \sqrt{h \varepsilon} \cdot \sum_{j=0}^{i-1} 2^{-j / 2} \quad\left(x \in D_{i}, x<1\right) .
$$

In addition,

$$
\begin{gathered}
K_{0}^{x} \text { and } K_{1}^{x+2^{-i}} \text { intersect the closed arc } \\
\widehat{A_{j}^{0} A_{j}^{1}}\left(x \in D_{i}, x<1, i \leq j \leq n\right) .
\end{gathered}
$$

We put $K_{0}^{0}=K_{0}$ and $K_{1}^{1}=K_{1}, C_{0}^{0}=M$. It is clear that (1)-(5) are satisfied for $i=0$. (As for (4), we interpret the empty sum in (4) as zero. Note also that $\left|O_{0} O\right|=\left|O_{1} O\right|=\sin (h / 2)<h$, where $O$ denotes the origin.)

Let $0 \leq i<n$ be given, and suppose that the points $C_{i}^{x}$ and the circles $K_{0}^{x}, K_{1}^{x}$ have been defined for the relevant values of $x$, and satisfy (1)-(5).

For every $x \in D_{i}, x<1$ we denote the intersection of $K_{0}^{x}$ and $\widehat{A_{i+1}^{0} A_{i+1}^{1}}$ by $C_{i+1}^{x}$. Similarly, for every $x \in D_{i}, x>0$ we denote the intersection of $K_{1}^{x}$ and $\widehat{A_{i+1}^{0} A_{i+1}^{1}}$ by $C_{i+1}^{x-2^{-i-1}}$. These points exist by $(5)$.

Let $x \in D_{i}, x>0$ be fixed. The point $Q=P_{x-2^{-i-1}}$ is the middle point of the arc $\widehat{P_{x-2^{-i}} P_{x}}$. Therefore, $Q$ is outside the circle $K_{1}^{x}$. The distance $d$ 
between the point $Q$ and the circle $K_{1}^{x}$ is less than the length of the arc $\widehat{Q P_{x}}$, which is $h \varepsilon / 2^{i+1}$. The distance $d^{\prime}$ between $C_{i+1}^{x-2^{-i-1}}$ and $Q$ is greater than $\varepsilon>3 \sqrt{h \varepsilon}>3 \sqrt{d}$ by $h<\varepsilon / 10$. In addition, we have $d^{\prime} \leq R+\varepsilon<2-\varepsilon<2-d$.

Therefore, we may apply (ii) of Lemma 2.1, and find that the circle $K_{1}^{x}$ can be rotated about the point $C_{i+1}^{x-2^{-i-1}}$ by an angle $\alpha$ in the negative direction such that $\sin \alpha<2 \sqrt{d}$ and the rotated copy of $K_{1}^{x}$, denoted by $K_{0}^{x-2^{-i-1}}$, contains the point $Q$. By the lemma, the distance between the centres of $K_{1}^{x}$ and $K_{0}^{x-2^{-i-1}}$ is less than

$$
4 \sqrt{d}<\frac{4}{\sqrt{2}} \cdot \sqrt{h \varepsilon} \cdot 2^{-i / 2}<3 \sqrt{h \varepsilon} \cdot 2^{-i / 2} .
$$

Consequently, the distance between the origin and the centre of $K_{0}^{x-2^{-i-1}}$ is less than $h+3 \sqrt{h \varepsilon} \cdot \sum_{j=0}^{i} 2^{-j / 2}$.

In this way we have defined the circles $K_{0}^{y}$ for every $y \in D_{i+1} \backslash D_{i}$.

In the same way, we obtain the circle $K_{1}^{x+2^{-i-1}}$ by rotating $K_{0}^{x}$ about the point $C_{i+1}^{x}$ by an angle $\alpha$ in the positive direction such that $\sin \alpha<$ $\sqrt{2 h \varepsilon} \cdot 2^{-i / 2}$, and the rotated copy of $K_{0}^{x}$, denoted by $K_{1}^{x+2^{-i-1}}$ contains the point $P_{x+2^{-i-1}}$. The same argument shows that the distance between the origin and the centre of $K_{1}^{x+2^{-i-1}}$ is less than $h+3 \sqrt{h \varepsilon} \cdot \sum_{j=0}^{i} 2^{-j / 2}$. This defines the circles $K_{1}^{y}$ for every $y \in D_{i+1} \backslash D_{i}$. It is clear that (4) holds for $i+1$ in place of $i$.

Since $\sum_{j=0}^{\infty} 2^{-j / 2}=2+\sqrt{2}<3.2$, the distance between the origin and any of the centres of $K_{0}^{y}$ and $K_{1}^{y}$ is less than

$$
h+9.6 \sqrt{h \varepsilon}<10 \sqrt{h \varepsilon}<\varepsilon
$$

for every $y \in D_{i+1}$.

Now we prove that (5) is satisfied with $i+1$ in place of $i$.

Let $x \in D_{i}, 0<x<1$ be fixed. Let $Q=P_{x-2^{-i-1}}$ and $K^{\prime}=K_{0}^{x-2^{-i-1}}$, $K^{\prime \prime}=K_{1}^{x+2^{-i-1}}$. We prove that $K^{\prime}$ and $K^{\prime \prime}$ intersect each $\operatorname{arc} \widehat{A_{j}^{0} A_{j}^{1}}(i<j \leq$ $n)$.

The point $C_{i+1}^{x-2^{-i-1}}$ belongs to the arc $\widehat{A_{i+1}^{0} A_{i+1}^{1}}$, and thus it is either inside or on the circle $K_{1}$. The point $Q$ is outside $K_{1}$, and thus the circle $K^{\prime}$ must intersect $K_{1}$ at a point $S_{1}$ belonging to the arc $\widehat{Q C_{i+1}^{x-2^{-i-1}}}$. Let $T_{1}$ be 
the other intersection of the circles $K^{\prime}$ and $K_{1}$. A similar argument shows that the circle $K^{\prime}$ intersects $K_{0}$ at a point $S_{0}$ belonging to the arc $\widehat{Q C_{i+1}^{x}}$. Let $T_{0}$ be the other intersection of the circles $K^{\prime}$ and $K_{0}$.

We show that $T_{0}$ and $T_{1}$ are outside the domain $H$. Since the distance between the centres of $K^{\prime}$ and $K_{0}$ is less than $h+\varepsilon<2 \varepsilon$, we have

$$
\left|S_{0} T_{0}\right| \geq 2 \sqrt{1-\varepsilon^{2}}>2-\varepsilon>R=\operatorname{diam} H .
$$

Thus $T_{0}$ and $S_{0}$ cannot be both in $H$. If $T_{0}$ is in $H$, then we have $S_{0} \notin H$, and then $S_{0}$ is on the subarc $\widehat{P_{0} M}$ of $K_{0}$. Therefore,

$$
\left|S_{0} T_{0}\right| \leq\left|T_{0} M\right|+\left|M S_{0}\right| \leq R+\varepsilon<2-\varepsilon<2 \sqrt{1-\varepsilon^{2}}
$$

which is impossible. Thus $T_{0} \notin H$. The same argument shows $T_{1} \notin H$.

If the point $M$ is outside the circle $K^{\prime}$ then, as $P_{1}$ is inside $K^{\prime}$, it follows that $S_{1} \notin H$. If $M$ is inside the circle $K^{\prime}$, then, as $P_{0}$ is outside $K^{\prime}$, it follows that $S_{0} \notin H$. If $M$ is on the circle $K^{\prime}$, then we have $S_{0}=S_{1}=M$. Let $\partial H$ denote the boundary of $H$. Then we have

$$
K^{\prime} \cap\left(K_{0} \cup K_{1}\right) \cap \partial H=\{S\},
$$

where $S$ is either $S_{0}$ or $S_{1}$ (or both if $S_{0}=S_{1}=M$ ).

Now $K^{\prime}$ must intersect the boundary of $H$ at two points. Let the intersection other than $S$ be $U$. Since $T_{0}, T_{1} \notin H$, the point $U$ must be on the arc

$\widehat{A_{n}^{0} A_{n}^{1}}$. Therefore, the subarc $\widehat{C_{i+1}^{x-2^{-i-1}} U}$ of $K^{\prime}$ is in $H$, and thus $K^{\prime}$ intersects each of the $\operatorname{arcs} \widehat{A_{j}^{0} A_{j}^{1}}$ for every $i<j \leq n$.

A similar argument shows that $K^{\prime \prime}$ also intersects each of the $\operatorname{arcs} \widehat{A_{j}^{0} A_{j}^{1}}$ for every $i<j \leq n$, and thus (5) is satisfied with $i+1$ in place of $i$.

This completes the construction of the points $C_{i}^{x}$ and circles $K_{1}^{x}, K_{0}^{x}$.

\section{Moving circular arcs}

Let $\sigma$ be a circular arc of radius 1 and of arc length less than 1.32. Then the diameter of the set $\sigma$ is less than $2 \sin (1.32 / 2)<1.227=R$. We describe a continuous motion of $\sigma$ starting from the subarc $\widehat{M S_{0}}$ of $K_{0}$ lying on the 
boundary of $L_{1}$. Its final position will be the subarc $\widehat{M S_{1}}$ of $K_{1}$ also on the boundary of $L_{1}$.

We choose a large $n$, and take the circles of the sprouting process. We use the notation of the previous section. The centre of $K_{i}^{x}$ is denoted by $O_{i}^{x}$ $(i=0,1)$.

First we rotate $\sigma$ about the point $O_{0}$ such that its lower endpoint becomes $A_{n}^{0}=C_{n}^{0}$. Then we rotate $\sigma$ about the point $C_{n}^{0}$ in the positive direction until it becomes a subarc of $K_{1}^{2^{-n}}$. Let $\alpha_{n}^{0}$ denote the angle of the rotation. Then we rotate $\sigma$ about the point $O_{1}^{2^{-n}}$ into $L_{0}$ with upper endpoint $P_{2^{-n}}$. Then we rotate $\sigma$ about the point $P_{2^{-n}}$ in the positive direction until it becomes a subarc of $K_{0}^{2^{-n}}$. Let $\beta_{n}^{2^{-n}}$ denote the angle of the rotation. Then we rotate $\sigma$ about the point $O_{0}^{2^{-n}}$ back into $L_{1}$ such that its lower endpoint becomes $C_{n}^{2^{-n}}$. Then we rotate $\sigma$ about the point $C_{n}^{2^{-n}}$ in the positive direction until it becomes a subarc of $K_{1}^{2 \cdot 2^{-n}}$. Let $\alpha_{n}^{2^{-n}}$ denote the angle of the rotation. Then we rotate $\sigma$ about the point $O_{1}^{2 \cdot 2^{-n}}$ into $L_{0}$ with upper endpoint $P_{2 \cdot 2^{-n}}$. Then we rotate $\sigma$ about the point $P_{2^{-n}}$ in the positive direction until it becomes a subarc of $K_{0}^{2 \cdot 2^{-n}}$. Let $\beta_{n}^{2 \cdot 2^{-n}}$ denote the angle of the rotation. Then we rotate $\sigma$ about the point $O_{0}^{2 \cdot 2^{n}}$ back into $L_{1}$ such that its lower endpoint becomes $C_{n}^{2 \cdot 2^{-n}}$. We continue this process until $\sigma$ becomes a subarc of $K_{0}^{\left(2^{n}-1\right) \cdot 2^{-n}}$ with lower endpoint $C_{n}^{\left(2^{n}-1\right) \cdot 2^{-n}}=A_{n}^{1}$. Then we rotate $\sigma$ about the point $A_{n}^{1}$ in the positive direction until it becomes a subarc of $K_{1}$. Let $\alpha_{n}^{\left(2^{n}-1\right) \cdot 2^{-n}}$ denote the angle of the rotation. Then we rotate $\sigma$ about the point $O_{1}$ until it reaches its final position $\widehat{M S_{1}}$.

Let $x \in D_{n}, x<1$. Let $H_{i}^{x}$ denote the horn shaped domain bounded by the subarc $\widehat{P_{x} C_{i}^{x}}$ of $K_{0}^{x}$, the subarc $\widehat{P_{x+2^{-i}} C_{i}^{x}}$ of $K_{1}^{x+2^{-i}}$, and the arc $\widehat{P_{x} P_{x+2^{-i}}}$. We put $\Delta(h)=H_{0}^{0}$. Then $\Delta(h)$ is the horn shaped domain bounded by the subarc $\overparen{P_{0} M}$ of $K_{0}, \widehat{P_{1} M}$ of $K_{1}$ and the arc $\overparen{P_{0} P_{1}}$. We put

$$
T_{n}(h)=\bigcup_{x \in D_{n}, x<1} H_{n}^{x}
$$

Our next aim is to estimate the area of $T_{n}(h) \backslash \Delta(h)$. The area (Lebesgue measure) of a set $A \subset \mathbb{R}^{2}$ will be denoted by $m(A)$.

If a point $P$ belongs to $T_{n}(h) \backslash \Delta(h)$, then there is a smallest $j>0$ such that $P \in H_{j}^{x}$ for some $x \in D_{j}, x<1$. If $j=i+1$, then

$$
P \in H_{i+1}^{x} \backslash \bigcup_{y \in D_{i}, y<1} H_{i}^{y}
$$




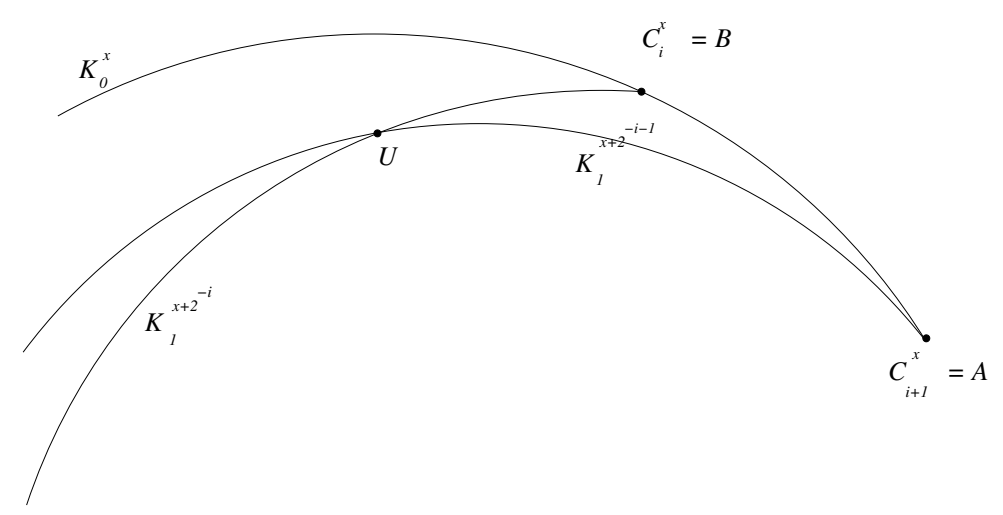

Figure 2: $H_{i+1}^{x} \backslash H_{i}^{x}$

for some $x \in D_{i+1}, x<1$. Now we have either $x \in D_{i}$ or $x-2^{-i-1} \in D_{i}$. In the first case $P \in H_{i+1}^{x} \backslash H_{i}^{x}$, while in the second case $P \in H_{i+1}^{x} \backslash H_{i}^{x-2^{-i-1}}$. We have proved the following:

$$
\begin{aligned}
T_{n}(h) \backslash \Delta(h) \subset & \bigcup_{i=0}^{n-1} \bigcup_{x \in D_{i}} \bigcup_{x<1}\left(H_{i+1}^{x} \backslash H_{i}^{x}\right) \cup \\
& \cup \bigcup_{i=0}^{n-1} \bigcup_{x \in D_{i+1} \backslash D_{i}}\left(H_{i+1}^{x} \backslash H_{i}^{x-2^{-i-1}}\right) .
\end{aligned}
$$

The set $H_{i+1}^{x} \backslash H_{i}^{x}$ is bounded by three subarcs of the circles $K_{0}^{x}, K_{1}^{x+2^{-i}}$ and $K_{1}^{x+2^{-i-1}}$ (see Figure 2). Let $U$ denote the intersection of $K_{1}^{x+2^{-i}}$ and $K_{1}^{x+2^{-i-1}}$ belonging to $L_{1}$. We want to apply Lemma 2.2 with the choice $K=K_{0}^{x}, A=C_{i+1}^{x}, B=C_{i}^{x}, \alpha=\alpha_{i+1}^{x}$ being the angle of rotation about $A$ mapping $K_{1}^{x+2^{-i-1}}$ onto $K_{0}^{x}$, and $\beta=\alpha_{i}^{x}$ being the angle of rotation about $B$ mapping $K_{1}^{x+2^{-i}}$ onto $K_{0}^{x}$. Now we check that (for $n$ large enough) the conditions of Lemma 2.2 are satisfied. First we need the following lemma (its proof will be given in Section 6).

Lemma 4.1. There exists a positive constant $c$ depending on $h$ and $\varepsilon$ but not on $n$ such that $\left|C_{i}^{x} C_{i+1}^{x}\right| \leq c / n$ and $\left|C_{i}^{x} C_{i+1}^{x+2^{-i-1}}\right| \leq c / n$ hold for every $n=1,2, \ldots, 0 \leq i<n$ and $x \in D_{i}, x<1$.

By this lemma, we have

$$
\eta=C_{i+1}^{x} O_{0}^{x} C_{i}^{x} \angle<2 c / n<1 / 5
$$


if $n>10 c$. Next we need the following estimates for $\alpha_{i}^{x}$.

Lemma 4.2. For every $1 \leq i \leq n$ and $x \in D_{i}, x<1$ we have

$$
0.9 \cdot \frac{h \varepsilon \cdot 2^{-i}}{t \cdot \sqrt{1-(t / 2)^{2}}}<\alpha_{i}^{x}<1.1 \cdot \frac{h \varepsilon \cdot 2^{-i}}{t \cdot \sqrt{1-(t / 2)^{2}}}
$$

where $t=\left|P_{x+2^{-i}} C_{i}^{x}\right|$.

We prove Lemma 4.2 in Section 6 .

Clearly, $\left|C_{i}^{x} C_{i+1}^{x}\right| \geq 1 / n$, and thus $\eta=C_{i+1}^{x} O_{0}^{x} C_{i}^{x} \angle>1 / n$. Since $t=t_{i}>$ $\varepsilon$ and $t \leq \varepsilon+R \leq 2-4 \varepsilon$, (9) gives

$$
\alpha_{i}^{x} \leq 1.1 \cdot \frac{h \varepsilon \cdot 2^{-i}}{\varepsilon \cdot \sqrt{1-(1-2 \varepsilon)^{2}}} \leq 1.1 \cdot \frac{h \cdot 2^{-i}}{\sqrt{2 \varepsilon}}<2^{-i} \cdot 10^{-3}<2^{-i},
$$

where we used that $\varepsilon<1 / 2$ and $h / \varepsilon<10^{-3}$. If $i>\log _{2} n$, then this gives $\alpha_{i}^{x}<1 / n<\eta$. Thus, when applying Lemma 2.2, the condition $\beta<\eta$ is satisfied if $i>\log _{2} n$. Finally, we prove that the condition $\alpha / \beta<3 / 4$ is satisfied if $i$ is large enough. We have, by (9),

$$
\alpha / \beta=\alpha_{i+1}^{x} / \alpha_{i}^{x}<\frac{1}{2} \cdot \frac{1.1}{0.9} \cdot \frac{t_{i} \cdot \sqrt{1-\left(t_{i} / 2\right)^{2}}}{t_{i+1} \cdot \sqrt{1-\left(t_{i+1} / 2\right)^{2}}},
$$

where $t_{i}=\left|P_{x+2^{-i}} C_{i}^{x}\right|$ and $t_{i+1}=\left|P_{x+2^{-i-1}} C_{i+1}^{x}\right|$. Clearly,

$$
\left|t_{i+1}-t_{i}\right| \leq\left|P_{x+2^{-i}} P_{x+2^{-i-1}}\right|+\left|C_{i}^{x} C_{i+1}^{x}\right|<2^{-i}+(c / n) .
$$

Since the function $t \mapsto t \cdot \sqrt{1-(t / 2)^{2}}$ is positive and uniformly continuous on the interval $[0,2-4 \varepsilon]$, there exists a positive number $v$ only depending on $\varepsilon$ such that

$$
\left(t \cdot \sqrt{1-(t / 2)^{2}}\right) /\left(t^{\prime} \cdot \sqrt{1-\left(t^{\prime} / 2\right)^{2}}\right)<1.1
$$

whenever $t, t^{\prime} \in[0,2-4 \varepsilon]$ and $\left|t-t^{\prime}\right|<v$. If $n>(1+c) / v$, and $i>\log _{2} n$, then (11) and $(12)$ imply $\alpha / \beta<(1 / 2) \cdot(1.1 / 0.9) \cdot 1.1<3 / 4$.

Therefore, assuming Lemmas 4.1 and 4.2 we can see that all conditions of Lemma 2.2 are satisfied provided that $n>\max (10 c,(1+c) / v)$ and $i>\log _{2} n$. Therefore, the circles $K_{1}^{x+2^{-i}}$ and $K_{1}^{x+2^{-i-1}}$ intersect at a point $U$ such that 
$\left|U C_{i+1}^{x}\right|<20 \eta<40 c / n$. Rotating the subarc $\widehat{U C_{i+1}^{x}}$ of $K_{1}^{x+2^{-i-1}}$ about the point $C_{i+1}^{x}$ in the negative direction by angle $\alpha_{i+1}^{x}$ we obtain a horn shaped domain $H^{\prime}$ which contains $H_{i+1}^{x} \backslash H_{i}^{x}$. Then

$$
m\left(H^{\prime}\right)=\left|U C_{i+1}^{x}\right|^{2} \cdot \alpha_{i+1}^{x} / 2<(40 c / n)^{2} \cdot 2^{-i}=1600 c^{2} \cdot 2^{-i} / n^{2}
$$

by 100 and Lemma 2.4 . We find that

$$
m\left(H_{i+1}^{x} \backslash H_{i}^{x}\right)<1600 c^{2} \cdot 2^{-i} / n^{2}
$$

if $n>\max (10 c,(1+c) / v)$ and $i>\log _{2} n$. A similar argument shows that if if $n>\max (10 c,(1+c) / v)$ and $i>\log _{2} n$, then

$$
m\left(H_{i+1}^{x} \backslash H_{i}^{x-2^{-i-1}}\right)<10000 c^{2} \cdot 2^{-i} / n^{2} .
$$

The only difference is that we have to apply Lemma 2.3 instead of Lemma 2.2. We omit the details. Since the cardinality of $D_{i}$ equals $2^{i}+1$, we obtain that the area of the set

$$
\bigcup_{i=\left\lceil\log _{2} n\right\rceil}^{n-1} \bigcup_{x \in D_{i}}\left(H_{x<1}^{x} \backslash H_{i+1}^{x}\right) \cup \bigcup_{i=\left\lceil\log _{2} n\right\rceil}^{n-1} \bigcup_{x \in D_{i+1} \backslash D_{i}}\left(H_{i+1}^{x} \backslash H_{i}^{x-2^{-i-1}}\right)
$$

is less than

$$
2 \cdot n \cdot 2^{i} \cdot 10000 c^{2} \cdot 2^{-i} / n^{2}=20000 c^{2} / n
$$

if $n>\max (10 c,(1+c) / v)$. If $i \leq \log _{2} n$, then for all $x \in D_{i}, x<1, H_{i}^{x}$ is contained in the disc of centre $M$ and radius $i R / n<2\left(\log _{2} n\right) / n$. Thus we have that the set

$$
\bigcup_{i=0}^{\left\lfloor\log _{2} n\right\rfloor} \bigcup_{x \in D_{i}}\left(H_{x<1}^{x} \backslash H_{i+1}^{x} \backslash H_{i}^{\left\lfloor\log _{2} n\right\rfloor} \bigcup_{i=0} \bigcup_{x \in D_{i+1} \backslash D_{i}}\left(H_{i+1}^{x} \backslash H_{i}^{x-2^{-i-1}}\right)\right.
$$

is contained in the disc of centre $M$ and radius less than $2\left(\left(\log _{2} n\right)+1\right) / n$. Thus the set $T_{n}(h) \backslash \Delta(h)$ can be covered by a disc of radius $2\left(\left(\log _{2} n\right)+1\right) / n$ and a set of area $20000 c^{2} / n$. Since $c$ does not depend on $n$ and $\left(\log _{2} n\right) / n \rightarrow 0$ as $n \rightarrow \infty$, we have proved the following.

Lemma 4.3. The area of the set $T_{n}(h) \backslash \Delta(h)$ tends to zero as $n \rightarrow \infty$. 


\section{Proof of Theorem 1.2}

Let $\sigma_{0}$ be a circular arc of radius 1 and of arc length less than 1.32, and let $\sigma_{1}$ be obtained from $\sigma_{0}$ by rotating it about one of its end points by a given angle $h \in(0, \pi)$. Let $\varepsilon>0$ be given. We have to prove that there is a continuous motion bringing $\sigma_{0}$ to $\sigma_{1}$ such that the area touched by the moving arc is less than $\varepsilon$. Let diam $\sigma_{0}$ denote the diameter of $\sigma_{0}$, then we have diam $\sigma_{0}<1.227$. We may assume that $\varepsilon<10^{-6}$. In the argument below we fix $\varepsilon$ with this property.

We shall use the notation of the previous two sections. We shall indicate the dependence of the objects on the angle $h$ whenever necessary. That is, $K_{0}(h)$ and $K_{1}(h)$ will denote the circles of radius 1 and centres $O_{0}=$ $(-\sin (h / 2), 0)$ and $O_{1}=(\sin (h / 2), 0)$, respectively, for every $h \in(0, \pi)$. The point with coordinates $(0, \cos (h / 2))$ is denoted by $M(h)$.

Let $\bar{K}_{i}(h)$ denote the disc bounded by $K_{i}(h)(i=0,1)$. The closures of the sets $\bar{K}_{0}(h) \backslash \bar{K}_{1}(h)$ and $\bar{K}_{1}(h) \backslash \bar{K}_{0}(h)$ are denoted by $L_{0}(h)$ and $L_{1}(h)$.

We may assume that $\sigma_{0}=\widehat{M(h) S_{0}(h)}$ and $\sigma_{1}=\widehat{M(h) S_{1}(h)}$, where $\widehat{M(h) S_{0}(h)}$ is a subarc of $K_{0}(h) \cap L_{1}(h)$ and $\widehat{M(h) S_{1}(h)}$ is a subarc of $K_{1}(h)$.

The circle of radius $\varepsilon$ and centre $M(h)$ intersects the lune $L_{0}(h)$ in the arc $\widehat{P_{0}(h) P_{1}(h)}$, where $P_{0}(h) \in K_{0}(h)$ and $P_{1}(h) \in K_{1}(h)$.

The intersection of $L_{0}(h)$ and the disc of centre $M(h)$ and radius $\varepsilon$ is denoted by $\Delta(h)$. Clearly, $m(\Delta(h))<\varepsilon^{2} \pi<\varepsilon / 2$.

Let $\Lambda$ denote the set of all positive numbers $\lambda$ having the following property: for every $0<h<\pi$ there is a set $\Sigma(h, \lambda)$ such that

$$
m(\Sigma(h, \lambda) \backslash \Delta(h))<\lambda \cdot h,
$$

and an arc can be moved continuously in the set $\Sigma(h, \lambda)$ with initial position $\widehat{M(h) S_{0}(h)}$ and final position $\widehat{M(h) S_{1}(h)}$.

If we rotate $\widehat{M(h) S_{0}(h)}$ about the point $M(h)$ in the positive direction by angle $h$, then the area of the set $\Sigma$ of points touched by the moving arc equals $\left(\operatorname{diam} \sigma_{0}\right)^{2} \cdot h / 2$ by Lemma 2.4 . Therefore, we have $\left(\operatorname{diam} \sigma_{0}\right)^{2} / 2 \in \Lambda$, and thus $\Lambda \neq \emptyset$.

Our aim is to show that if $\lambda \in \Lambda$, then $\frac{q+3}{4} \cdot \lambda \in \Lambda$ (see Lemma 5.3 below), where $0<q<1$ is a constant depending only on $\varepsilon$. 
We will need the following lemma about the sum of the angles $\beta_{n}^{x}$ of rotations around the points $P_{x}$.

Lemma 5.1. There exists a positive constant $q<1$ depending only on $\varepsilon$ such that

$$
\sum_{x \in D_{n}, 0<x<1} \beta_{n}^{x} \leq q \cdot h
$$

In Section 6 we prove that the statement of Lemma 5.1 is true with $q=1-\varepsilon^{4}$.

We shall use the notation

$$
U(A, \eta)=\left\{x \in \mathbb{R}^{2}: \operatorname{dist}(x, A)<\eta\right\}
$$

for every $A \subset \mathbb{R}^{2}$ and $\eta>0$.

Lemma 5.2. For every $0<h<\varepsilon^{2} / 100$ and $\lambda \in \Lambda$ there is a set $\Sigma^{\prime}(h, \lambda)$ such that

$$
m\left(\Sigma^{\prime}(h, \lambda) \backslash U(\Delta(h), \sqrt{h})\right)<\frac{1+q}{2} \cdot \lambda \cdot h,
$$

and an arc can be moved continuously in the set $\Sigma^{\prime}(h, \lambda)$ with initial position $\widehat{M(h) S_{0}(h)}$ and final position $\widehat{M(h) S_{1}(h)}$.

Proof. Let $0<h<\varepsilon^{2} / 100$ and $\lambda \in \Lambda$ be fixed. In the argument below we shall suppress the reference to $h$; that is, we shall write $K_{0}$ instead of $K_{0}(h)$ etc.

We apply the sprouting process as described in Section 3, and construct the set $T_{n}$ as described in Section 4. By Lemma 4.3, we can choose an $n$ such that

$$
m\left(T_{n} \backslash \Delta(h)\right)<\frac{1-q}{2} \cdot \lambda \cdot h
$$

holds. The movement of the arc consists of rotating about the points $C_{n}^{x}$ and $P_{x}$ alternately, and moving the arc along some circles in between. The angle of the rotation about the point $P_{x}$ equals $\beta_{n}^{x}$. The aim of this rotation is to bring the arc from $K_{1}^{x}$ to $K_{0}^{x}$.

Now we shall replace the set touched by the arc during the rotation about the point $P_{x}$ by a smaller set using the condition $\lambda \in \Lambda$. We fix $x \in D_{n}$, $0<x<1$, and write $\beta$ in place of $\beta_{n}^{x}$. By definition we can move an arc 
continuously in the set $\Sigma(\beta, \lambda)$ such that its initial position is $\widehat{M(\beta) S_{0}(\beta)}$ and its final position is $\widehat{M(\beta) S_{1}(\beta)}$.

Let $\tau_{x}$ denote the isometry mapping $O_{0}(\beta)$ into $O_{0}^{x}$ and $O_{1}(\beta)$ into $O_{1}^{x}$. Note that $\left|O_{0}(\beta) O_{1}(\beta)\right|=\left|O_{0}^{x} O_{1}^{x}\right|=2 \sin (\beta / 2)$. The isometry $\tau_{x}$ is obtained by a reflection about the $y$ axis followed by a suitable rotation by an angle close to $\varepsilon$ which maps the point $M(\beta)$ into $P_{x}$. Then $\tau_{x}$ is an isometry mapping the circle $K_{1}(\beta)$ into $K_{1}^{x}$ and the circle $K_{0}(\beta)$ into $K_{0}^{x}$. It is clear that we can move an arc continuously in the set $\tau_{x}(\Sigma(\beta, \lambda))$ such that its initial position is a subarc of $K_{1}^{x} \cap L_{0}(h)$ and its final position is a subarc of $K_{0}^{x} \cap L_{0}(h)$.

Let $\Sigma^{\prime}(h, \lambda)$ denote the set

$$
T_{n} \cup \bigcup_{x \in D_{n}, 0<x<1} \tau_{x}\left(\Sigma\left(\beta_{n}^{x}, \lambda\right)\right) .
$$

We prove that $\Sigma^{\prime}(h, \lambda)$ satisfies the condition of the lemma. It follows from the construction that we can move an arc continuously in $\Sigma^{\prime}(h, \lambda)$ from the initial position $\widehat{M S_{0}}$ to the final position $\widehat{M S_{1}}$. In order to prove (14) first we show that

$$
\tau_{x}\left(\Delta\left(\beta_{n}^{x}\right)\right) \subset U(\Delta(h), \sqrt{h})
$$

for every $x \in D_{n}, 0<x<1$. The set $\tau_{x}\left(\Delta\left(\beta_{n}^{x}\right)\right)$ is bounded by subarcs of $K_{1}^{x}, K_{0}^{x}$ and of the circle with centre $P_{x}$ and radius $\varepsilon$ (see Figure 3 ). Let $K_{0}^{\prime}$ denote the circle of radius 1 going through the points $P_{x}$ and $M=M(h)$ obtained from $K_{0}$ by rotating it about the point $M$ in the positive direction by an angle $<h$. It is clear that $K_{0}^{\prime}$ intersects the arc $\widehat{A_{n}^{0} A_{n}^{1}}$. Let $C$ denote the point of intersection. The circle $K_{0}^{x}$ also intersects the arc $\widehat{A_{n}^{0} A_{n}^{1}}$ at the point $C_{n}^{x}$. Since $\left|C_{n}^{x} C\right|<\left|A_{n}^{0} A_{n}^{1}\right|<R \cdot h<2 h$ and $\left|P_{x} C\right|>\varepsilon>3 \sqrt{2 h}$, $\left|P_{x} C\right|<R+\varepsilon=2-4 \varepsilon<2-2 h$, we may apply Lemma 2.1 and find that $K_{0}^{x}$ is obtained from $K_{0}^{\prime}$ by rotating it about the point $P_{x}$ by an angle $\alpha$ such that $\sin \alpha<2 \sqrt{2 h}$. Thus $\alpha<4 \sqrt{2 h}$. The same argument shows that $K_{1}^{x}$ is obtained from $K_{0}^{\prime}$ by rotating it about the point $P_{x}$ by an angle $<4 \sqrt{2 h}$.

Since $\left|P_{x} M\right|=\varepsilon$, we can see that every point $A$ of $\tau_{x}\left(\Delta\left(\beta_{n}^{x}\right)\right)$ can be obtained from a point $B$ of the subarc $\widehat{P_{x} M}$ of $K_{0}^{\prime}$ by rotating it about the point $P_{x}$ by an angle $<4 \sqrt{2 h}$. Then $|A B|<\varepsilon \cdot 4 \sqrt{2 h}<6 \varepsilon \sqrt{h}<\sqrt{h}$. Since $B \in \Delta(h)$, this proves $(17)$.

We have

$$
m\left(\Sigma\left(\beta_{n}^{x}, \lambda\right) \backslash \Delta\left(\beta_{n}^{x}\right)\right)<\lambda \cdot \beta_{n}^{x}
$$




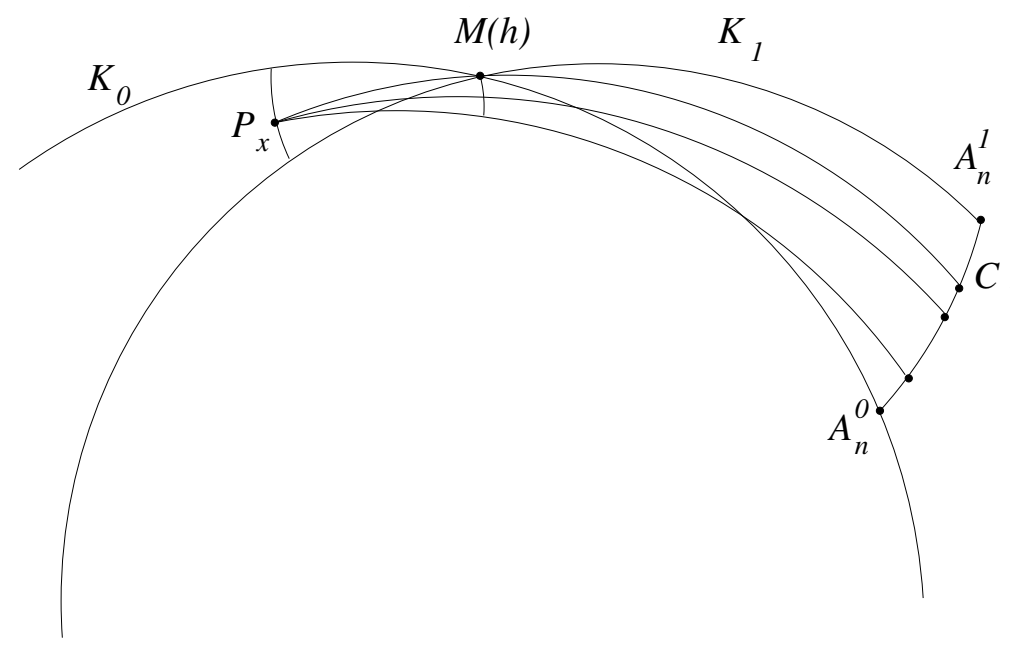

Figure 3: Proof of Lemma 5.2

for every $x \in D_{n}, 0<x<1$ by the definition of $\lambda$. Then it follows from (15), (16) and (17) that the area of the set $\Sigma^{\prime}(h, \lambda) \backslash U(\Delta(h), \sqrt{h})$ is less than

$$
\left(\frac{1-q}{2} \cdot \lambda \cdot h\right)+\sum_{x \in D_{n}, 0<x<1} \lambda \cdot \beta_{n}^{x} .
$$

Since $\sum_{x \in D_{n}, x>0} \beta_{n}^{x} \leq q \cdot h$ by Lemma 5.1, we find that the area in question is less than

$$
\frac{1-q}{2} \cdot \lambda \cdot h+q \cdot \lambda \cdot h=\frac{1+q}{2} \cdot \lambda \cdot h .
$$

This completes the proof of Lemma 5.2 .

Lemma 5.3. For every $\lambda \in \Lambda$ we have $\frac{q+3}{4} \cdot \lambda \in \Lambda$.

Proof. Let $h \in(0, \pi)$ and $\lambda \in \Lambda$ be arbitrary. Let $G$ be an open set containing $\Delta(h)$ such that $m(G \backslash \Delta(h))<\frac{1-q}{4} \cdot \lambda \cdot h$. Since $\Delta(h)$ is compact, there is an $\eta>0$ such that $U(\Delta(h), \eta) \subset G$. We fix a positive number $\delta$ such that $\delta<\min \left(\varepsilon^{2} / 100, \eta^{2}\right)$.

We consider the subarc $\widehat{O_{0} O_{1}}$ of the circle of centre $M(h)$ and radius 1 . We select points of division $O_{0}=X_{0}, X_{1}, \ldots, X_{k}=O_{1}$ on the arc $\widetilde{O_{0} O_{1}}$ such that each of the angles $h_{i}=X_{i} M(h) X_{i-1} \angle(i=1, \ldots, k)$ is less than $\delta$ (see Figure (4). Note that $h_{1}+\ldots+h_{k}=h$. 


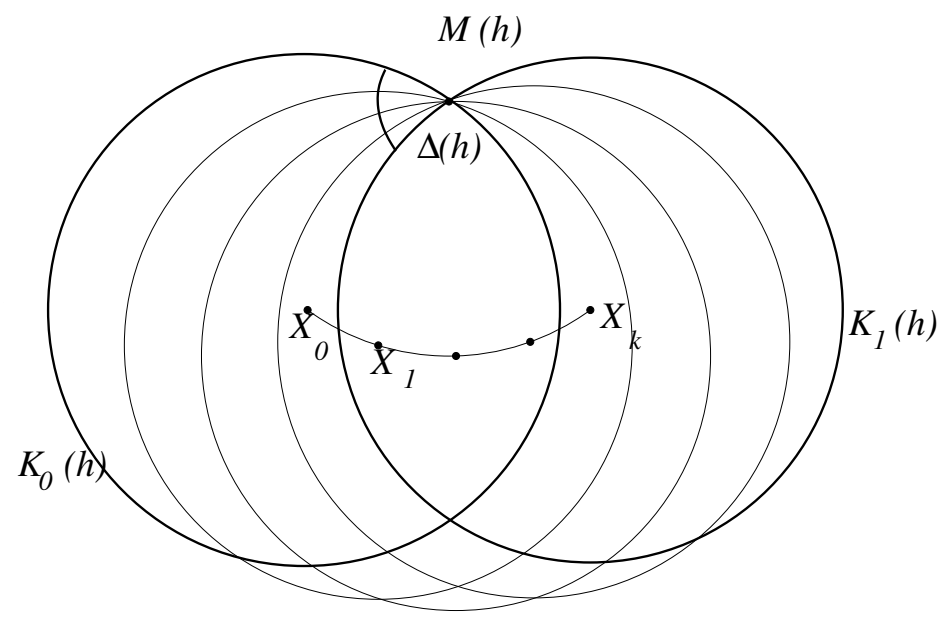

Figure 4: Proof of Lemma 5.3

The circle of centre $X_{i}$ and radius 1 will be denoted by $N_{i}(i=0, \ldots, m)$. Thus $N_{0}=K_{0}(h)$ and $N_{k}=K_{1}(h)$.

For every $i=1, \ldots, k$ there is an isometry (in fact, a rotation by an angle less than $h) \kappa_{i}$ mapping $K_{0}\left(h_{i}\right)$ onto $N_{i-1}$ and $K_{1}\left(h_{i}\right)$ onto $N_{i}$. It is easy to see that $\kappa_{i}\left(\Delta\left(h_{i}\right)\right) \subset \Delta(h)$ for every $i=1, \ldots, k$. We put

$$
\Sigma=\sum_{i=1}^{k} \kappa_{i}\left(\Sigma^{\prime}\left(h_{i}, \lambda\right)\right)
$$

where the set $\Sigma^{\prime}\left(h_{i}, \lambda\right)$ is given by Lemma 5.2. It is clear that we can move an arc continuously in the set $\Sigma$ with initial position $\widehat{M(h) S_{0}(h)}$ and final position $\widehat{M(h) S_{1}(h)}$. Since $\sqrt{h_{i}}<\sqrt{\delta}<\eta$ for every $i$, it follows from (14) that

$$
m\left(\kappa_{i}\left(\Sigma^{\prime}\left(h_{i}, \lambda\right)\right) \backslash U(\Delta(h), \eta)\right)<\frac{1+q}{2} \cdot \lambda \cdot h_{i}
$$

for every $i$. Therefore, we have

$$
m(\Sigma \backslash U(\Delta(h), \eta))<\sum_{i=1}^{k} \frac{1+q}{2} \cdot \lambda \cdot h_{i}=\frac{1+q}{2} \cdot \lambda \cdot h .
$$

Hence, by $m(U(\Delta(h), \eta) \backslash \Delta(h))<\frac{1-q}{4} \cdot \lambda \cdot h$ we obtain

$$
m(\Sigma \backslash \Delta(h))<\left(\frac{1-q}{4}+\frac{1+q}{2}\right) \cdot \lambda \cdot h=\frac{q+3}{4} \cdot \lambda \cdot h .
$$


Since $0<h<\pi$ was arbitrary, this means, by the definition of $\Lambda$ that $\frac{q+3}{4} \cdot \lambda \in \Lambda$, which completes the proof of the lemma.

In possession of Lemma 5.3 we prove Theorem 1.2 as follows. Let $\sigma_{0}$ and $\sigma_{1}$ be as in Theorem 1.2 . We may assume that the angle $h$ of the rotation bringing $\sigma_{0}$ onto $\sigma_{1}$ is less than $\pi$. Then we may also assume that $\sigma_{0}=\widehat{M(h) S_{0}(h)}$ and $\sigma_{1}=\widehat{M(h) S_{1}(h)}$.

As $\Lambda \neq \emptyset$, we can choose a positive number $\lambda_{0} \in \Lambda$. Then, by Lemma 5.3. $\lambda_{n}=\left(\frac{q+3}{4}\right)^{n} \cdot \lambda_{0} \in \Lambda$ for every $n$. Since $0<q<1$, thus $0<\frac{q+3}{4}<1$ and $\lambda_{n} \rightarrow 0$ as $n \rightarrow \infty$, hence we can choose a $\lambda \in \Lambda$ such that $\lambda<\varepsilon /(2 \pi)$.

By the definition of $\Lambda$ this means that there is a set $\Sigma(h, \lambda)$ such that $m(\Sigma(h, \lambda) \backslash \Delta(h))<\lambda \cdot h<\varepsilon / 2$, and an arc can be moved continuously in the set $\Sigma(h, \lambda)$ with initial position $\widehat{M(h) S_{0}(h)}$ and final position $\widehat{M(h) S_{1}(h)}$. Since $m(\Delta(h))<\varepsilon / 2$, we have $m(\Sigma(h, \lambda))<\varepsilon$, and thus Theorem 1.2 is proved, subject to the Lemmas whose proofs were postponed.

\section{Proof of the lemmas}

Proof of Lemma 2.1. We shall use the following notation. Let $A$ be the closest point of $K$ to $P$, and let $B$ be the point obtained by reflecting $A$ about $O$. Then the points $B, O, P, A$ are collinear, and $|P A|=d$. Let $R$ be the point of the segment $O A$ such that $|R A|=2 d$. Let $C, D \in K$ be such that the segment $C D$ is perpendicular to $O A$ and contains $R$. We assume that $C$ and $Q$ belong to the same semicircle with endpoints $B$ and $A$. Then we have

$$
|R C|=\sqrt{|R A| \cdot|R B|}=\sqrt{2 d \cdot(2-2 d)}=2 \sqrt{d-d^{2}} .
$$

Now we turn to the proof of the lemma.

(i) Suppose that $P$ is in the interior of $K$. Then we have

$$
|P C|=\sqrt{|P R|^{2}+|R C|^{2}}=\sqrt{d^{2}+4 \cdot\left(d-d^{2}\right)}<2 \sqrt{d} .
$$

Since $|Q P| \geq 2 \sqrt{d}$, it follows that $Q$ belongs to the arc $\overparen{C B}$. By $(18)$ we have

$$
|B C|^{2}=|B R|^{2}+|R C|^{2}=(2-2 d)^{2}+4\left(d-d^{2}\right)=4-4 d<(2-d)^{2}=|B P|^{2},
$$




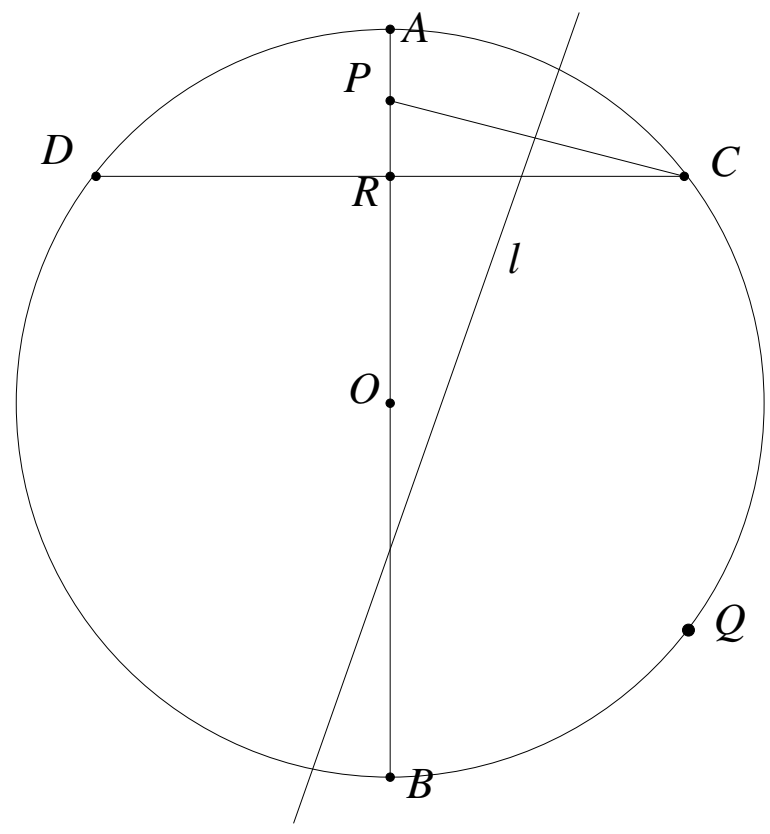

Figure 5: Case (i)

and thus $|B P|>|B C|$. Now we prove $|Q P|>|Q C|$.

Let $\ell$ denote the perpendicular bisector of the segment $P C$. Since $|B P|>$ $|B C|$ and $|D P|<|D C|$, it follows that $\ell$ intersects the arc $\overparen{B D}$. It is clear that $\ell$ intersects the arc $\overparen{A C}$ as well, and thus the arc $\overparen{B C}$ is disjoint from $\ell$. Then every point of $\overparen{B C}$ is closer to $C$ then to $P$. In particular, $|Q P|>|Q C|$. Thus the circle of radius $|Q P|$ and centre $Q$ intersects the arc $\overparen{A C}$ at a unique point $P^{\prime}$ such that the orientation of the triangle $\Delta_{P Q P^{\prime}}$ is positive. Let $\alpha=P^{\prime} Q P \angle$. It is clear that $\alpha<\pi / 2$, and rotating $K$ about the point $Q$ in the positive direction by angle $\alpha$, the circle obtained will contain $P$. We show $\sin \alpha<2 \sqrt{d}$. Indeed, we have

$$
\alpha<C Q P \angle<C Q D \angle=C B D \angle=\frac{1}{2} \cdot C O D \angle=C O R \angle,
$$

and thus $\sin \alpha<\sin C O R \angle<2 \sqrt{d}$.

(ii) Suppose that $P$ is outside the circle $K$. Then we have

$$
|P C|=\sqrt{(3 d)^{2}+\left(4 d-4 d^{2}\right)}<\sqrt{9 d}=3 \sqrt{d} .
$$




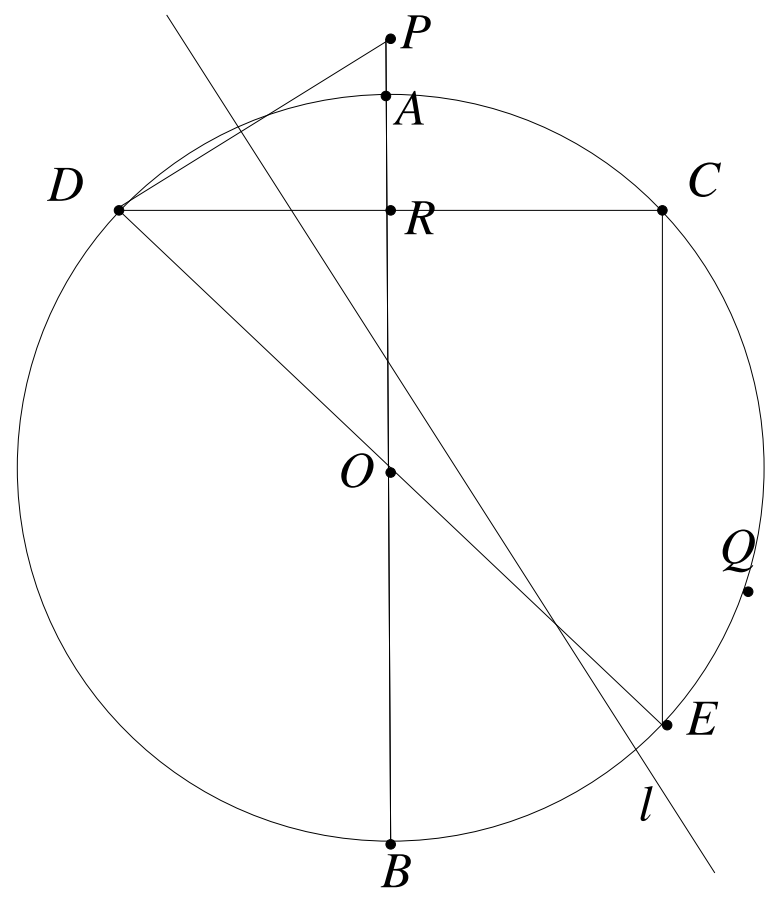

Figure 6: Case (ii)

Since $|Q P| \geq 3 \sqrt{d}$, it follows that $Q$ belongs to the arc $\overparen{C B}$. Let $E$ be the point obtained by reflecting $D$ about $O$. Then $\triangle_{D E C}$ is a right triangle, and $C E$ is parallel to $A B$. Let $S$ be the point obtained by reflecting $R$ about $O$. Then we have

$$
|P S|=|P R|+2 \cdot|R O|=3 d+2(1-2 d)=2-d,
$$

and thus

$$
|P E|^{2}=|P S|^{2}+|S E|^{2}=(2-d)^{2}+4 \cdot\left(d-d^{2}\right)=4-3 d^{2} .
$$

This gives

$$
2-d<|P E|<2=|D E| .
$$

Then, by $|P Q| \leq 2-d$ it follows that $Q$ belongs to the arc $\overparen{C E}$. We prove that $|Q P|<|Q D|$. Let $\ell$ denote the perpendicular bisector of the segment $D P$. Since $|P E|<|D E|$ and $|P B|>|D B|$, it follows that $\ell$ intersects the arc $\overparen{B E}$. Similarly, $|A P|=d<3 d<|D P|$ implies that $\ell$ intersects the arc 


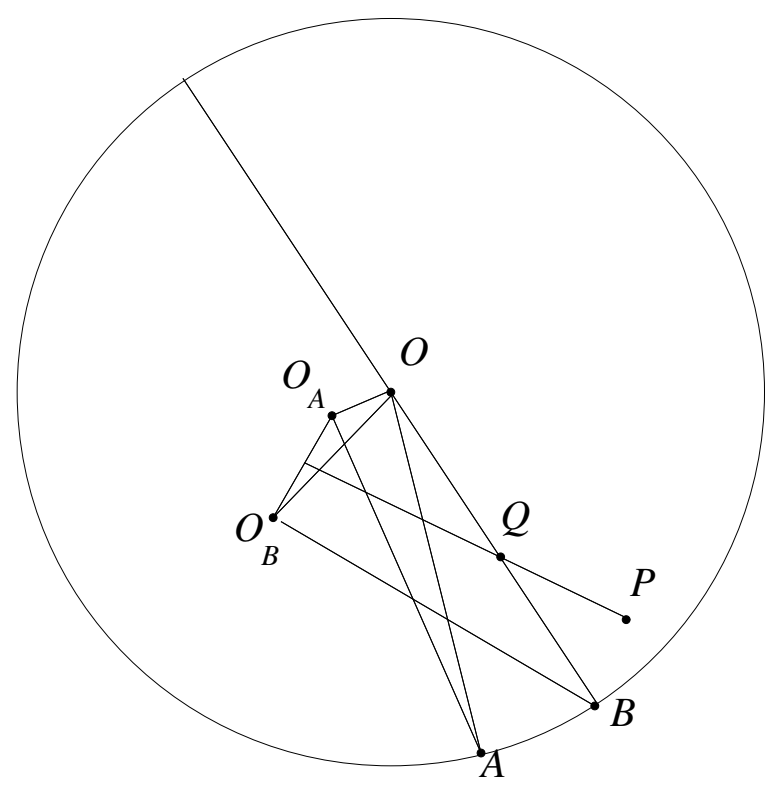

Figure 7: Lemma 2.2

$\overparen{D A}$ as well. Therefore, the arc $\overparen{C E}$ is disjoint from $\ell$, and thus every point of $\overparen{C E}$ is closer to $P$ then to $D$. This proves $|Q P|<|Q D|$. Therefore, the circle of radius $|Q P|$ and centre $Q$ intersects the arc $\overparen{D A}$ at a unique point $P^{\prime}$ such that the orientation of the triangle $\triangle_{P Q P^{\prime}}$ is negative. Let $\alpha=P Q P^{\prime} \angle$. It is clear that $\alpha<\pi / 2$, and rotating $K$ about the point $Q$ in the negative direction by angle $\alpha$, the circle obtained will contain $P$.

It is easy to check that the tangent line of $K$ at the point $C$ does not separate the points $P$ and $Q$. Therefore, we have $P Q P^{\prime} \angle \leq C Q D \angle$, and thus

$$
\alpha=P Q P^{\prime} \angle \leq C Q D \angle=C B D \angle=\frac{1}{2} \cdot C O D \angle=C O R \angle .
$$

This gives $\sin \alpha<\sin C O R \angle<2 \sqrt{d}$.

We have proved that $\sin \alpha<2 \sqrt{d}$ in both cases. Let $O^{\prime}$ denote the centre of $K^{\prime}$. Then $\triangle_{O Q O^{\prime}}$ is an isosceles triangle such that $|O P|=\left|O^{\prime} P\right|=1$ and $O P O^{\prime} \angle=\alpha$. Thus $\left|O O^{\prime}\right|=2 \sin (\alpha / 2)<2 \sin \alpha<4 \sqrt{d}$.

Proof of Lemma 2.2. Let $O_{A}$ and $O_{B}$ denote the centres of $K_{A}$ and $K_{B}$, 
respectively. Then we have $\left|O_{A} O\right|=2 \sin (\alpha / 2)<2 \sin (\beta / 2)=\left|O_{B} O\right|$, and

$$
\begin{aligned}
O_{A} O O_{B} \angle & =O_{A} O A \angle+A O B \angle-B O O_{B} \angle= \\
& =\left(\frac{\pi}{2}-\frac{\alpha}{2}\right)+\eta-\left(\frac{\pi}{2}-\frac{\beta}{2}\right)=\eta+\frac{\beta-\alpha}{2} .
\end{aligned}
$$

Since $\eta+(\beta-\alpha) / 2>0$, we obtain that the orientation of the triangle $O O_{A} O_{B}$ is positive. If $O O_{A} O_{B} \angle=\gamma$, then

$$
O_{A} O_{B} O \angle=\pi-\gamma-\eta-\frac{\beta-\alpha}{2}=\pi-\left(\gamma+\eta+\frac{\beta-\alpha}{2}\right) .
$$

Therefore, by the sine law applied to the triangle $\Delta_{O O_{A} O_{B}}$ we obtain

$$
\frac{\sin \left(\gamma+\eta+\frac{\beta-\alpha}{2}\right)}{\sin \gamma}=\frac{\sin (\alpha / 2)}{\sin (\beta / 2)} .
$$

It is easy to check that $5 \cdot \sin (1 / 5)>15 / 16$. Since the function $(\sin x) / x$ is decreasing in $(0, \pi / 2)$, it follows that $(\sin x) / x>15 / 16$ for every $0<x<1 / 5$. In particular, we have $\sin \beta / 2>15 \beta / 32$, and thus the right hand side of (19) is less than

$$
\frac{\alpha / 2}{15 \beta / 32}=\frac{\alpha}{\beta} \cdot \frac{16}{15}<\frac{3}{4} \cdot \frac{16}{15}=\frac{4}{5} .
$$

The left hand side of 19 equals

$$
\cos \left(\eta+\frac{\beta-\alpha}{2}\right)+\cot \gamma \cdot \sin \left(\eta+\frac{\beta-\alpha}{2}\right) .
$$

Since

$$
\cos \left(\eta+\frac{\beta-\alpha}{2}\right)>\cos 2 \eta \geq \cos 2 / 5>9 / 10
$$

it follows that

$$
\cot \gamma \cdot \sin \left(\eta+\frac{\beta-\alpha}{2}\right)<\frac{4}{5}-\frac{9}{10}=-\frac{1}{10}
$$

This implies $\cot \gamma<0, \pi / 2<\gamma<\pi$, and

$$
\cot (\pi-\gamma) \cdot \sin \left(\eta+\frac{\beta-\alpha}{2}\right)>\frac{1}{10}
$$

Since $\sin (\eta+(\beta-\alpha) / 2)<\sin 2 \eta<2 \eta$, we obtain $\tan (\pi-\gamma)<20 \eta$. 
The intersection $K_{A} \cap K_{B}$ consists of two points, each on the perpendicular bisector of the segment $O_{A} O_{B}$. Let $P$ be the one which is on the same side of the line $O_{A} O_{B}$ as the points $A$ and $B$. We have

$$
O_{A} O B \angle=O_{A} O O_{B} \angle+O_{B} O B \angle=\eta+\frac{\beta-\alpha}{2}+\frac{\pi-\beta}{2}=\frac{\pi}{2}+\eta-\frac{\alpha}{2} .
$$

Then, by $\alpha<\eta$, we obtain that $O_{A} O B \angle$ is an obtuse angle. Therefore, we have $\left|B O_{A}\right|>|B O|=1=\left|B O_{B}\right|$. Since $\left|O O_{A}\right|<\left|O O_{B}\right|$, it follows that the perpendicular bisector of the segment $O_{A} O_{B}$ intersects the segment $O B$ at a point $Q$. Clearly, $\left|O_{B} Q\right|<\left|O_{B} B\right|=1=\left|O_{B} P\right|$, and thus $P$ and $O_{B}$ are separated by the line $O B$. Therefore, $O_{A} O P \angle>O_{A} O B \angle>\pi / 2$, and then $|O P|<\left|O_{A} P\right|=1$. This proves that $P$ is inside $K$.

Since $\left|A O_{A}\right|=\left|P O_{A}\right|=1$, the triangle $\triangle_{A P O_{A}}$ is isosceles. If $P O_{A} A \angle=$ $\delta$, then

$$
\gamma=O O_{A} O_{B} \angle=\left(P O_{A} O_{B} \angle\right)+\left(O O_{A} A \angle\right)-\delta<2 \cdot(\pi / 2)-\delta=\pi-\delta,
$$

and thus $\delta<\pi-\gamma$. Therefore, we have

$$
|A P|=2 \sin (\delta / 2)<\tan \delta<\tan (\pi-\gamma)<20 \eta
$$

which completes the proof of the lemma.

Proof of Lemma 2.3. The proof is similar to that of Lemma 2.2 (see Figure 8). It is easy to check that $O_{A} O O_{B} \angle=\eta-(\beta-\alpha) / 2$, and that the orientation of the triangle $O O_{A} O_{B}$ is positive. Let $O O_{A} O_{B} \angle=\gamma$. Then

$$
O_{A} O_{B} O \angle=\pi-\left(\gamma+\eta-\frac{\beta-\alpha}{2}\right)
$$

and from this we obtain $\pi / 2<\gamma<\pi$ and $\tan (\pi-\gamma)<20 \eta$ the same way as in the proof of Lemma 2.2 .

Since $1=\left|B O_{B}\right|>\left|B O_{A}\right|$, the perpendicular bisector of $\overline{O_{A} O_{B}}$ intersects the segment $\overline{O_{B} B}$ at a point $Q$. Then it follows from $\left|O_{B} Q\right|<\left|O_{B} B\right|=1$ that $P$ and $O_{A}$ are separated by the line $\overline{O_{B} B}$.

We have

$$
O O_{A} P \angle=2 \pi-\left(\gamma+\left(P O_{A} O_{B} \angle\right)\right)>2 \pi-\left(\pi+\frac{\pi}{2}\right)=\frac{\pi}{2},
$$




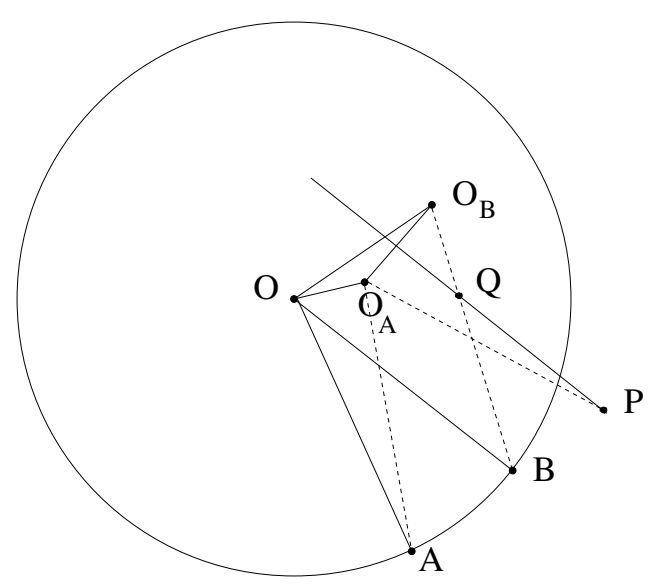

Figure 8: Lemma 2.3

and thus $|P O|>\left|P O_{A}\right|=1$. This proves that $P$ is outside $K$.

Since $\left|O_{A} P\right|=\left|O_{B} P\right|=1$, the triangle $\Delta_{O_{A} P O_{B}}$ is isosceles. Let $\zeta$ denote the angle $O_{A} P O_{B} \angle$. Since the angle $\gamma$ is obtuse, we have $\left|O_{A} O_{B}\right|<\left|O O_{B}\right|$, and thus $\zeta<\beta$.

Let $P O_{A} A \angle=\delta$. Then

$$
\delta=2 \pi-\gamma-\frac{\pi-\alpha}{2}-\frac{\pi-\zeta}{2}<\pi-\gamma+\eta .
$$

Hence

$$
\begin{aligned}
|A P| & =2 \sin (\delta / 2)<\tan \delta<\tan (\pi-\gamma+\eta)=\frac{\tan (\pi-\gamma)+\tan \eta}{1-\tan (\pi-\gamma) \cdot \tan \eta}< \\
& <\frac{20 \eta+2 \eta}{1-40 \eta^{2}}<50 \eta
\end{aligned}
$$

which completes the proof of the lemma.

Proof of Lemma 2.4. If $\alpha=2 \pi / n$, then the statement follows from the fact that the disc of radius $|A B|$ can be decomposed into $n$ congruent copies of $H$. Therefore, the statement is also true if $\alpha$ is a rational multiple of $\pi$. The general case follows by approximating $\alpha / \pi$ from below and from above by rational numbers. 
Lemma 6.1. Let $a \leq b \leq c$ be the sides of a triangle, and let $\alpha$ be its angle opposite to the side $a$. Then $\alpha<2 a / c$.

Proof. Since $\alpha$ is the smallest angle of the triangle, we have $\alpha<\pi / 2$, and thus $\alpha<2 \sin \alpha$. If $\gamma$ is the angle opposite to the side $c$, then we have $\sin \alpha / \sin \gamma=a / c, \sin \alpha \leq a / c$ and $\alpha<2 a / c$.

In the sequel we shall use the following notation. Let $h, \varepsilon$ be positive numbers satisfying $\varepsilon<10^{-6}$ and $h \leq \varepsilon / 10^{3}$. Let $K_{0}$ and $K_{1}$ be the circles of radius 1 and centres $(-\sin (h / 2), 0)$ and $(\sin (h / 2), 0)$, respectively. The point with coordinates $(0, \cos (h / 2))$ will be denoted by $M$. Thus $M \in K_{0} \cap K_{1}$.

Let $\bar{K}_{i}$ denote the disc bounded by $K_{i}(i=0,1)$. The closures of the sets $\bar{K}_{0} \backslash \bar{K}_{1}$ and $\bar{K}_{1} \backslash \bar{K}_{0}$ are denoted by $L_{0}$ and $L_{1}$.

The circle of radius $\varepsilon$ and centre $M$ intersects the lune $L_{0}$ in the arc $\widehat{P_{0} P_{1}}$, where $P_{0} \in K_{0}$ and $P_{1} \in K_{1}$.

Proof of Lemma 2.5. First we show that

$$
\left|\left(P_{0} M O \angle\right)-(\pi / 2)\right|<h+\varepsilon
$$

The triangle $\Delta_{P_{0} O_{0} M}$ is isosceles, and $\left|P_{0} M\right|=\varepsilon$. If $\alpha_{0}=P_{0} O_{0} M \angle$, then $\alpha_{0}<2 \varepsilon$, by Lemma 6.1. Since $P_{0} M O \angle=\left(\left(\pi-\alpha_{0}\right) / 2\right)+(h / 2),(20)$ follows. A similar argument gives

$$
\left|P_{1} M O \angle-(\pi / 2)\right|<h+\varepsilon .
$$

The inequalities (20) and (21) imply that the angle between the $x$ axis and any line connecting $M$ to a point of $\widehat{P_{0} P_{1}}$ is less than $h+\varepsilon$. Therefore, the angle between the $y$ axis and any tangent line of $\widehat{P_{0} P_{1}}$ is less than $h+\varepsilon$. Now, if a line intersects the arc $\widehat{P}_{0} P_{1}$ at two points, then it is parallel to a tangent line of $\widehat{P_{0} P_{1}}$, and then the statement of the lemma follows.

Proof of Lemma 2.6. First we show that the orientation of the triangle $\Delta_{Q^{\prime \prime} O^{\prime} C}$ is positive. Let $D$ denote the circle of centre $O_{0}$ and radius $1.5 \varepsilon$. Since $\left|O^{\prime} O\right|<\varepsilon$ and $\left|O O_{0}\right|<h / 2$, we have $O^{\prime} \in D$. Let $\ell$ denote the line going through $Q^{\prime \prime}$, tangent to the disc $D$ at a point $E$, and such that the orientation of the triangle $\Delta_{Q^{\prime \prime} O_{0} E}$ is positive. Let $M^{\prime} \in K_{0}$ be such that the 


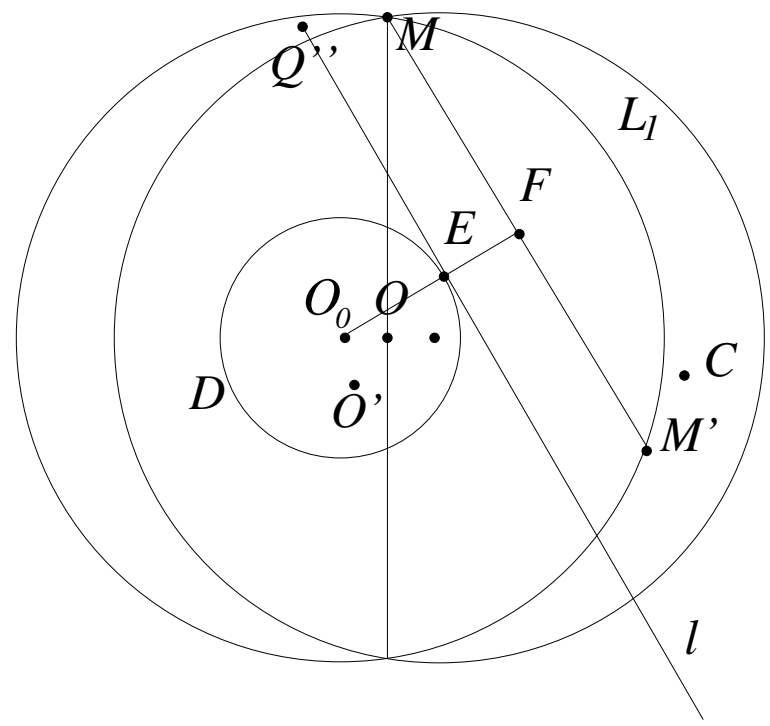

Figure 9: Orientation of $\Delta_{Q^{\prime \prime} O^{\prime} C}$

line $\overline{M M^{\prime}}$ is parallel to $\ell$. The line $\overline{M M^{\prime}}$ is obtained from $\ell$ by translating it by a vector of length $<\varepsilon$. Since the distance between the point $O_{0}$ and $\ell$ is $1.5 \varepsilon$, the distance between the point $O_{0}$ and $\overline{M M^{\prime}}$ is at most $2.5 \varepsilon$.

Let $F$ denote the middle point of the segment $M M^{\prime}$. Then $\left|O_{0} F\right| \leq 2.5 \varepsilon$, $|M F|>1-2.5 \varepsilon$ and $\left|M M^{\prime}\right|=2|M F|>2-5 \varepsilon$. Since $C \in L_{1}$ and $|M C| \leq$ $2-5 \varepsilon$, it follows that the orientation of the triangle $\Delta_{M M^{\prime} C}$ is positive (see Figure 91. Thus the orientation of the triangle $\Delta_{Q^{\prime \prime} O^{\prime} C}$ is also positive.

The point $Q^{\prime \prime}$ is inside the circle $K^{\prime}$, and the distance $d$ between the point $Q^{\prime \prime}$ and the circle $K^{\prime}$ is at most the length of the arc $\widehat{Q^{\prime} Q^{\prime \prime}}$, which is at most $h \varepsilon$. Since the distance $d^{\prime}$ between $C$ and $Q^{\prime \prime}$ is at least $\varepsilon>2 \sqrt{h \varepsilon}>2 \sqrt{d}$, we may apply (i) of Lemma 2.1, and find that $\sin \alpha<2 \sqrt{d}<\varepsilon / 2$. Thus $\alpha<\varepsilon$.

Let the point $\tilde{Q}$ be selected such that $Q^{\prime}, O^{\prime}, O, \tilde{Q}$ are the vertices of a parallelogram. Then

$$
|\tilde{Q} M| \leq\left|\tilde{Q} Q^{\prime}\right|+\left|Q^{\prime} M\right|=\left|O^{\prime} O\right|+\left|Q^{\prime} M\right|<2 \varepsilon .
$$

Thus, by Lemma 6.1 applied to the triangle $\Delta_{\tilde{Q} O M}$ we find that $\tilde{Q} O M \angle<4 \varepsilon$; that is, the angle $\gamma$ of the line $Q^{\prime} O^{\prime}$ and the $y$ axis is less than $4 \varepsilon$.

Let $t=\left|Q^{\prime \prime} C\right|=\left|\rho\left(Q^{\prime \prime}\right) C\right|$. Then $t<\left|Q^{\prime \prime} M\right|+|M C|<\varepsilon+2-5 \varepsilon<2$, 
and thus

$$
\left|Q^{\prime \prime} \rho\left(Q^{\prime \prime}\right)\right|=t \cdot 2 \sin (\alpha / 2)<2 \cdot 2 \sin (\alpha / 2)<2 \alpha<2 \varepsilon .
$$

Therefore, $\left|Q^{\prime} \rho\left(Q^{\prime \prime}\right)\right| \leq\left|Q^{\prime} Q^{\prime \prime}\right|+\left|Q^{\prime \prime} \rho\left(Q^{\prime \prime}\right)\right|<3 \varepsilon$, and then $Q^{\prime} O^{\prime} \rho\left(Q^{\prime \prime}\right) \angle<3 \varepsilon$. If the angle between the $x$ axis and the line $\overline{Q^{\prime} \rho\left(Q^{\prime \prime}\right)}$ is $\zeta$, then

$$
\zeta \leq \gamma+\left|\left(O^{\prime} Q^{\prime} \rho\left(Q^{\prime \prime}\right) \angle\right)-(\pi / 2)\right|<4 \varepsilon+\frac{1}{2} \cdot Q^{\prime} O^{\prime} \rho\left(Q^{\prime \prime}\right) \angle<6 \varepsilon,
$$

which proves (iii).

Lemma 6.2. There exists an $n_{0}$ depending on $h$ but not on $n$ such that $|X Y|<6 h$ for every $n \geq n_{0}, 0 \leq i<n, X \in \widehat{A_{i}^{0} A_{i}^{1}}$ and $Y \in \widehat{A_{i+1}^{0} A_{i+1}^{1}}$.

Proof. Since the function $\arccos x$ is uniformly continuous on the interval $[0,1]$, there is a $u>0$ such that $|\arccos x-\arccos y|<h$ whenever $x, y \in[0,1]$ and $|x-y| \leq u$. We show that $n_{0}=2 / u$ satisfies the requirement. Suppose $n \geq n_{0}$.

For every $X \in \widehat{A_{i}^{0} A_{i}^{1}}$ the distance $\left|X A_{i}^{0}\right|$ is less than the length of the $\operatorname{arc} \widehat{A_{i}^{0} A_{i}^{1}}$ which is less than $2 h$. Similarly, we have $\left|Y A_{i+1}^{0}\right|<2 h$ for every $Y \in \widehat{A_{i+1}^{0} A_{i+1}^{1}}$.

Let $\alpha_{i}, \alpha_{i+1}, \delta_{i}$ denote the angles $O_{0} M A_{i}^{0} \angle, O_{0} M A_{i+1}^{0} \angle, A_{i+1}^{0} M A_{i}^{0} \angle$, respectively. Then

$$
\delta_{i}=\alpha_{i}-\alpha_{i+1}=\arccos \left(r_{i} / 2\right)-\arccos \left(r_{i+1} / 2\right)<h,
$$

as $\left|r_{i+1}-r_{i}\right|=R / n<2 / n<u$. Since $A_{i}^{0} O_{0} A_{i+1}^{0} \angle=2 \delta_{i}$, (23) implies $\left|A_{i}^{0} A_{i+1}^{0}\right|=2 \sin \delta_{i}<2 \delta_{i}<2 h$, and hence,

$$
|X Y| \leq\left|X A_{i}^{0}\right|+\left|A_{i}^{0} A_{i+1}^{0}\right|+\left|Y A_{i+1}^{0}\right|<6 h .
$$

Proof of Lemma 4.1. Let $n_{0}$ be as in Lemma 6.2. We show that $c=$ $\max \left(2 n_{0}, 8 / \varepsilon\right)$ satisfies the requirement. If $n \leq n_{0}$ then $\left|C_{i}^{x} C_{i+1}^{x}\right| \leq 2 \leq c / n$ and $\left|C_{i}^{x} C_{i+1}^{x+2^{-i-1}}\right| \leq 2 \leq c / n$ for every $i$ and $x$. Therefore, we may assume that $n>n_{0}$. 


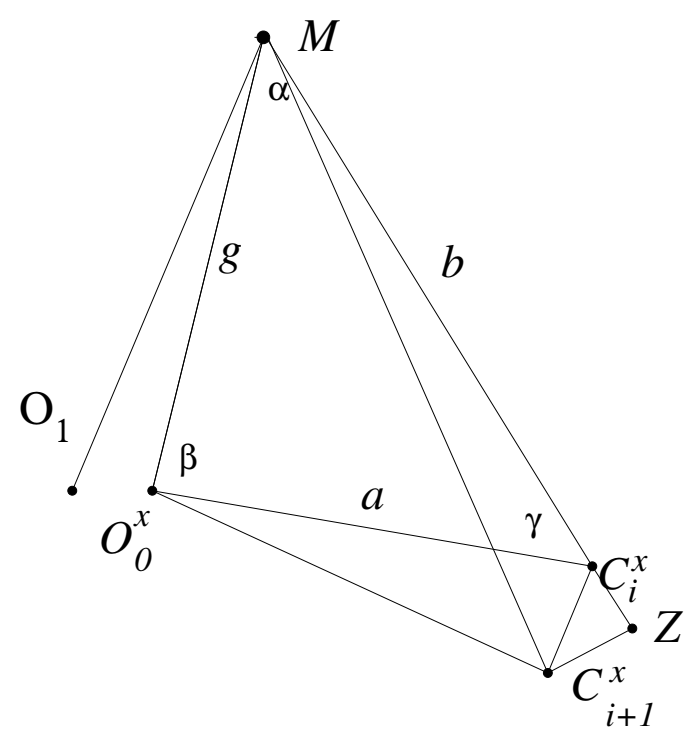

Figure 10: Lemma 4.1

We put $\left|O_{0}^{x} C_{i}^{x}\right|=a,\left|C_{i}^{x} M\right|=b$ and $\left|M O_{0}^{x}\right|=g$. Note that $a=1$ by $C_{i}^{x} \in K_{0}^{x}$, and $b=r_{i} \leq 2-5 \varepsilon$. Let $C_{i}^{x} M O_{0}^{x} \angle=\alpha, M O_{0}^{x} C_{i}^{x} \angle=\beta$, $O_{0}^{x} C_{i}^{x} M \angle=\gamma$. Our first aim is to show $\gamma>\varepsilon$.

Since $\left|O_{0}^{x} O\right|<\varepsilon$ and $\left|O O_{1}\right|<h / 2$, we have $\left|O_{0}^{x} O_{1}\right|<2 \varepsilon$ and $g=\left|O_{0}^{x} M\right|>$ $1-2 \varepsilon$. Since $\left|O_{1} M\right|=1$, we find $O_{0}^{x} M O_{1} \angle<4 \varepsilon$ by Lemma 6.1. We also have $O_{1} M C_{i}^{x} \angle<\pi / 2$, since $\overline{O_{1} M}$ is a ray of $K_{1}$ and $C_{i}^{x}$ is either inside or on the circle $K_{1}$. Therefore, we obtain $\alpha<(\pi / 2)+4 \varepsilon$. If $b<1-2 \varepsilon$, then $b$ is the shortest side of the triangle $\Delta_{O_{0}^{x} C_{i}^{x} M}$. Then, in this case, $\beta<\pi / 3$ and $\gamma=\pi-\alpha-\beta>(\pi / 6)-4 \varepsilon>\varepsilon$.

Now suppose $b \geq 1-2 \varepsilon$. Then we have, by $a=1$ and $b \leq 2-5 \varepsilon$,

$$
\begin{aligned}
1-\cos \gamma & =1-\frac{a^{2}+b^{2}-g^{2}}{2 a b}=\frac{g^{2}-(a-b)^{2}}{2 a b} \geq \frac{(1-2 \varepsilon)^{2}-(1-5 \varepsilon)^{2}}{2 a b}= \\
& \geq \frac{(3 \varepsilon)(2-7 \varepsilon)}{4}>\varepsilon .
\end{aligned}
$$

Therefore, we obtain $\gamma>1-\cos \gamma>\varepsilon$.

Let $Z \in \widehat{A_{i+1}^{0} A_{i+1}^{1}}$ be such that $M, C_{i}^{x}$ and $Z$ are collinear. Note that $\left|C_{i+1}^{x} C_{i}^{x}\right|<6 h$ by Lemma 6.2 and $n>n_{0}$. Since the triangle $\Delta_{O_{0}^{x} C_{i+1}^{x} C_{i}^{x}}$ is 
isosceles and $\left|O_{0}^{x} C_{i+1}^{x}\right|=\left|O_{0}^{x} C_{i}^{x}\right|=1$, we have $C_{i+1}^{x} O_{0}^{x} C_{i}^{x} \angle<12 h$ by Lemma 6.1. Then $O_{0}^{x} C_{i}^{x} C_{i+1}^{x} \angle>(\pi / 2)-6 h$, and

$$
C_{i+1}^{x} C_{i}^{x} Z \angle<\pi-((\pi / 2)-6 h)-\varepsilon<(\pi / 2)-(\varepsilon / 2) .
$$

Since $C_{i+1}^{x} Z C_{i}^{x} \angle<\pi / 2$, we obtain $C_{i}^{x} C_{i+1}^{x} Z \angle>\varepsilon / 2$. Therefore,

$$
\begin{aligned}
\frac{2}{n}>\frac{R}{n} & =\left|C_{i}^{x} Z\right| \geq\left|C_{i+1}^{x} C_{i}^{x}\right| \cdot \sin \left(C_{i}^{x} C_{i+1}^{x} Z \angle\right)>\left|C_{i+1}^{x} C_{i}^{x}\right| \cdot \sin (\varepsilon / 2)> \\
& >\left|C_{i+1}^{x} C_{i}^{x}\right| \cdot \frac{\varepsilon}{4} .
\end{aligned}
$$

This proves $\left|C_{i+1}^{x} C_{i}^{x}\right|<8 /(\varepsilon n) \leq c / n$.

The inequality $\left|C_{i}^{x} C_{i+1}^{x+2^{-i-1}}\right| \leq c / n$ can be proved similarly; we have to replace the point $O_{0}^{x}$ by $O_{1}^{x+2^{-i}}$, and use the fact that $K_{1}^{x+2^{-i}}$ contains the points $C_{i}^{x}$ and $C_{i+1}^{x+2^{-i-1}}$.

Proof of Lemma 4.2. We shall use the abbreviations $P=P_{x}, P^{\prime}=P_{x+2^{-i}}$, $O^{\prime}=O_{1}^{x+2^{-i}}, C=C_{i}^{x}$ and $\alpha=\alpha_{i}^{x}$ (see Figure 11). Also, we denote by $\rho$ the rotation about the point $C$ mapping $K_{1}^{x+2^{-i}}$ onto $K_{0}^{x}$. Since $P^{\prime}$ and $C$ lie on the circle $K_{1}^{x+2^{-i}}$ of centre $O^{\prime}$, we have $\left|O^{\prime} P^{\prime}\right|=\left|O^{\prime} C\right|=1$, and thus the triangle $\Delta_{P^{\prime} O^{\prime} C}$ is isosceles. Let $A$ denote the middle point of the segment $\overline{P^{\prime} C}$. Then triangle $\Delta_{P^{\prime} O^{\prime} A}$ has a right angle at the vertex $A$.

We have $\left|P^{\prime} M\right|=\varepsilon$ and $\left|O^{\prime} O\right|<\varepsilon$ by (6). Let the point $Q$ be selected such that $O^{\prime}, O, Q, P^{\prime}$ are the vertices of a parallelogram. Then

$$
|Q M| \leq\left|Q P^{\prime}\right|+\left|P^{\prime} M\right|=\left|O^{\prime} O\right|+\left|P^{\prime} M\right|<2 \varepsilon .
$$

Thus, by Lemma 6.1 applied to the triangle $\Delta_{M O Q}$ we find that the angle between the $y$ axis and the segment $\overline{O^{\prime} P^{\prime}}$ is less than $4 \varepsilon$.

We denote the angle $P^{\prime} O^{\prime} A \angle$ by $\phi$. Clearly, $\phi=\arcsin (t / 2)$.

By Lemma 2.5, the angle between the $y$ axis and the segment $\overline{P P^{\prime}}$ is less than $2 \varepsilon$. Therefore, if we add the angles at the vertex $P^{\prime}$ then we obtain

$$
\left|\left(\frac{\pi}{2}-\phi\right)+\left(\rho\left(P^{\prime}\right) P^{\prime} C \angle\right)+\left(P P^{\prime} \rho\left(P^{\prime}\right) \angle\right)-\pi\right|<6 \varepsilon .
$$

We have $\left|P^{\prime} C\right|=\left|\rho\left(P^{\prime}\right) C\right|=t$, as $\rho$ is a rotation about the point $C$. Thus the triangle $\Delta_{P^{\prime} C \rho\left(P^{\prime}\right)}$ is isosceles, and $\rho\left(P^{\prime}\right) P^{\prime} C \angle=(\pi-\alpha) / 2$. If we denote 


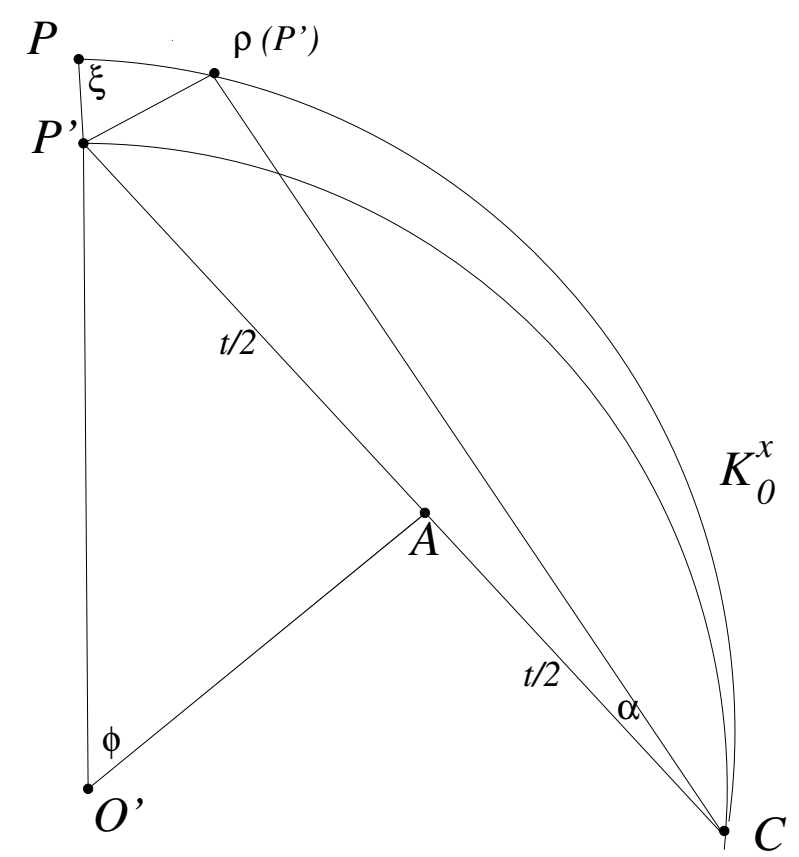

Figure 11: Lemma 4.2 
the angle $P P^{\prime} \rho\left(P^{\prime}\right) \angle$ by $\psi$, then (24) gives $|\psi-\phi-(\alpha / 2)|<6 \varepsilon$. Now we have $\alpha<\varepsilon$ by (i) of Lemma 2.6, and thus $|\psi-\arcsin (t / 2)|<7 \varepsilon$. Therefore, we have $|\cos (\arcsin (t / 2))-\cos \psi|<7 \varepsilon$; that is,

$$
\left|\cos \psi-\sqrt{1-(t / 2)^{2}}\right|<7 \varepsilon .
$$

Since $t \leq R+\varepsilon<2-4 \varepsilon$, we have

$$
\sqrt{1-(t / 2)^{2}}>\sqrt{1-(1-2 \varepsilon)^{2}}=\sqrt{4 \varepsilon-4 \varepsilon^{2}}>\sqrt{\varepsilon}>10^{3} \varepsilon
$$

by $\varepsilon<10^{-6}$. Then 25 gives $\cos \psi>900 \varepsilon$. We also have

$$
0.99<1-\frac{7}{10^{3}}<\frac{\cos \psi}{\sqrt{1-(t / 2)^{2}}}<1+\frac{7}{10^{3}}<1.01
$$

The angle between the $x$ axis and segment $\overline{P \rho\left(P^{\prime}\right)}$ is less than $6 \varepsilon$ by (ii) of Lemma 2.6. If $\xi$ denotes the angle $P^{\prime} P \rho\left(P^{\prime}\right) \angle$, then this implies $|\xi-(\pi / 2)|<$ $8 \varepsilon$ and $|\cot \xi|<10 \varepsilon$ by $\varepsilon<10^{-6}$.

Now we apply the sine law for the triangle $\Delta_{P P^{\prime} \rho\left(P^{\prime}\right)}$. Since $\left|P^{\prime} \rho\left(P^{\prime}\right)\right|=$ $2 t \sin (\alpha / 2)$, we obtain

$$
\frac{\left|P P^{\prime}\right|}{2 t \sin (\alpha / 2)}=\frac{\sin (\pi-\xi-\psi)}{\sin \xi}=\frac{\sin (\psi+\xi)}{\sin \xi}=\cos \psi+\cot \xi \cdot \sin \psi
$$

By $\cos \psi>900 \varepsilon$ and $|\cot \xi|<10 \varepsilon$ we find that

$$
0.95 \cos \psi<\cos \psi+\cot \xi \cdot \sin \psi<1.02 \cos \psi \text {. }
$$

The length of the arc $\widehat{P P^{\prime}}$ equals $h \varepsilon \cdot 2^{-i}$, and thus $\left|P P^{\prime}\right|=2 \varepsilon \sin \left(h \cdot 2^{-i-1}\right)$. This gives

$$
0.95 h \varepsilon \cdot 2^{-i}<\left|P P^{\prime}\right|<h \varepsilon \cdot 2^{-i} .
$$

Therefore, by (26) and (27) we obtain

$$
\begin{aligned}
\alpha & >2 \sin (\alpha / 2)=\frac{\left|P P^{\prime}\right|}{t(\cos \psi+\cot \xi \cdot \sin \psi)} \geq \frac{0.95 \cdot h \varepsilon \cdot 2^{-i}}{1.02 \cdot t \cdot \cos \psi}>0.93 \frac{h \varepsilon \cdot 2^{-i}}{t \cdot \cos \psi}> \\
& >0.9 \frac{h \varepsilon \cdot 2^{-i}}{t \cdot \sqrt{1-(t / 2)^{2}}}
\end{aligned}
$$


and

$$
\begin{aligned}
\alpha & <1.01 \cdot 2 \sin (\alpha / 2)=\frac{1.01 \cdot\left|P P^{\prime}\right|}{t(\cos \psi+\cot \xi \cdot \sin \psi)}<\frac{1.01}{0.95} \cdot \frac{h \varepsilon \cdot 2^{-i}}{t \cdot \cos \psi}< \\
& <1.07 \cdot \frac{h \varepsilon \cdot 2^{-i}}{t \cdot \cos \psi}<1.1 \cdot \frac{h \varepsilon \cdot 2^{-i}}{t \cdot \sqrt{1-(t / 2)^{2}}}
\end{aligned}
$$

which proves (9).

Proof of Lemma 5.1. We shall prove that the statement holds with $q=$ $1-\varepsilon^{4}$.

For all $x \in D_{n}, x>0$, let $C_{n}^{x} M C_{n}^{x-2^{-n}} \angle=\left(\beta_{n}^{x}\right)^{\prime}$. Then we have

$$
\sum_{x \in D_{n}, x>0}\left(\beta_{n}^{x}\right)^{\prime}=A_{n}^{0} M A_{n}^{1} \angle=h .
$$

Therefore, it is enough to prove that there is a positive constant $q<1$ depending only on $\varepsilon$ such that for all $x \in D_{n}, 0<x<1, \beta_{n}^{x} \leq q \cdot\left(\beta_{n}^{x}\right)^{\prime}$.

Fix $x \in D_{x}, 0<x<1$. We shall use the abbreviations $K=K_{0}^{x}, L=$ $K_{1}^{x}, P=P^{x}, A=C_{n}^{x}, B=C_{n}^{x-2^{-n}}, \beta=\beta_{n}^{x}$, and $\beta^{\prime}=\left(\beta_{n}^{x}\right)^{\prime}$. Then we have that $K$ contains $P$ and $A, L$ contains $P$ and $B$. Let $\rho$ denote the rotation about the point $P$ by angle $\beta$ in the positive direction. Then $\rho(L)=K$. Put $B^{\prime}=\rho(B) \in K, b=\left|B B^{\prime}\right|, s=|B P|=\left|B^{\prime} P\right|$, then $B P B^{\prime} \angle=\beta$ and $b=2 s \cdot \sin (\beta / 2)$.

It is easy to check that $\beta / 2<\varepsilon$, so we have

$$
b=2 s \sin \left(\frac{\beta}{2}\right)>2 s \cdot \frac{\beta}{2} \cdot \frac{\sin \varepsilon}{\varepsilon}>s \cdot \beta \cdot\left(1-\frac{\varepsilon^{2}}{6}\right)
$$

and

$$
\beta<\frac{b}{s} \cdot \frac{1}{1-\left(\varepsilon^{2} / 6\right)}<\frac{b}{s} \cdot\left(1+\varepsilon^{2}\right)
$$

Thus we have

$$
\frac{\beta}{\beta^{\prime}}<\frac{b}{\beta^{\prime}} \cdot \frac{1}{s}\left(1+\varepsilon^{2}\right)
$$

We shall estimate $b / \beta^{\prime}$ from above and $s$ from below. First we estimate $s$. 
Put $\delta=B M P \angle=N M P \angle+B M N \angle$. If the $y$ coordinate of $P$ is greater than or equal to the $y$ coordinate of $M$ (which is $\cos (h / 2)$ ), then $N M P \angle>\pi / 2$. Otherwise the tangent line at $P$ of the circle with centre $M$ and radius $\varepsilon$ intersects the $y$ axis at a point $Q$ with $y$ coordinate less than $\cos (h / 2)$. Then

$$
N M P \angle=Q M P \angle=\pi-\pi / 2-P Q M \angle \geq \pi / 2-h-\varepsilon
$$

by lemma 2.5 .

The point $B$ is on the arc $\widehat{A_{n}^{0} A_{n}^{1}}$ lying in the lune $L_{1}$. This implies $B M O_{0} \angle \geq A_{n}^{0} M O_{0} \angle$. Since the triangle $\Delta_{O_{0} A_{n}^{0} M}$ is isosceles with $\left|O_{0} M\right|=$ $\left|O_{0} A_{n}^{0}\right|=1$ and $\left|M A_{n}^{0}\right|=R$, we have

$$
B M O_{0} \angle \geq A_{n}^{0} M O_{0} \angle=\frac{\pi}{2}-\arcsin (R / 2) .
$$

Thus

$$
B M N \angle=B M O_{0} \angle-N M O_{0} \angle \geq \frac{\pi}{2}-\arcsin (R / 2)-(h / 2) .
$$

Therefore, we have the estimate

$$
\delta \geq \pi-\arcsin (R / 2)-(3 h / 2)-\varepsilon \geq \pi-\arcsin (R / 2)-2 \varepsilon
$$

by $\varepsilon \geq 2 h$. Then

$$
\begin{aligned}
\cos \delta & \leq \cos (\pi-\arcsin (R / 2)-2 \varepsilon)= \\
& =-\sqrt{1-\left(R^{2} / 4\right)} \cdot \cos (2 \varepsilon)+(R / 2) \cdot \sin (2 \varepsilon) \leq \\
& \leq-\sqrt{1-\left(R^{2} / 4\right)}\left(1-2 \varepsilon^{2}\right)+R \varepsilon \leq \\
& \leq-\sqrt{1-\left(R^{2} / 4\right)}+4 \varepsilon
\end{aligned}
$$

by $1 \leq R<2$. Now we apply the cosine law for $\Delta_{P B M}$ :

$$
s^{2}=R^{2}+\varepsilon^{2}-2 R \varepsilon \cos \delta \geq R^{2}+\varepsilon^{2}+2 R \varepsilon \sqrt{1-\left(R^{2} / 4\right)}-8 R \varepsilon^{2} .
$$

Using $1 \leq R<2$ again, we have

$$
s^{2} \geq R^{2}+R \varepsilon \sqrt{4-R^{2}}-15 \varepsilon^{2} \geq\left(R+(\varepsilon / 2) \cdot \sqrt{4-R^{2}}\right)^{2}-16 \varepsilon^{2} .
$$


Thus

$$
s-\left(R+(\varepsilon / 2) \cdot \sqrt{4-R^{2}}\right) \geq \frac{-16 \varepsilon^{2}}{s+R+(\varepsilon / 2) \cdot \sqrt{4-R^{2}}} \geq \frac{-16 \varepsilon^{2}}{2}=-8 \varepsilon^{2},
$$

where we estimated the denominator by using $s \geq R \geq 1$. We get

$$
s \geq R+(\varepsilon / 2) \cdot \sqrt{4-R^{2}}-8 \varepsilon^{2} .
$$

Now we estimate $b / \beta^{\prime}$ from above. Put $a=|A B|, d=\left|A B^{\prime}\right|, t=|P A|$. Applying the cosine low for $\Delta_{P A M}$, we have

$$
t^{2}=R^{2}+\varepsilon^{2}-2 R \varepsilon \cos \left(\delta+\beta^{\prime}\right)
$$

Thus

$$
t-s=\frac{t^{2}-s^{2}}{t+s}=\frac{2 R \varepsilon\left(\cos (\delta)-\cos \left(\delta+\beta^{\prime}\right)\right)}{t+s} \leq \frac{2 R \varepsilon \beta^{\prime}}{2 R}=\varepsilon \beta^{\prime},
$$

where we used $t \geq s \geq R$ and the Lipschitz continuity of the function $\cos x$.

Now we estimate $d$. The points $P, B^{\prime}$, and $A$ lie on the circle $K$ of radius 1 , and $|P A|=t,\left|P B^{\prime}\right|=s$, thus we get

$$
d<\widehat{A B^{\prime}}=\overparen{P A}-\widehat{P B^{\prime}}=2 \arcsin \left(\frac{t}{2}\right)-2 \arcsin \left(\frac{s}{2}\right)
$$

Applying the mean value theorem for the function $\arcsin (x)$ and using (30), we get

$$
d<2 \arcsin \left(\frac{t}{2}\right)-2 \arcsin \left(\frac{s}{2}\right) \leq 2\left(\frac{t}{2}-\frac{s}{2}\right) \cdot \frac{1}{\sqrt{1-\frac{t^{2}}{4}}} \leq \frac{2 \varepsilon \beta^{\prime}}{\sqrt{4-t^{2}}}
$$

Put $\phi=B A B^{\prime} \angle=B A P \angle+P A B^{\prime} \angle$. Since $P A B^{\prime} \angle$ is smaller than the angle $\psi$ of the secant $P A$ and the tangent line of the circle $K$ at $A$, and $\psi=\arcsin (t / 2)$, we have that $P A B^{\prime} \angle \leq \arcsin (t / 2)$. On the other hand, one can easily check that $B A P \angle<B A M \angle$, and since $\triangle_{B A M}$ is isosceles, $B A M \angle<\pi / 2$. Thus $\phi \leq(\pi / 2)+\arcsin (t / 2)$ and

$$
\cos \phi \geq \cos ((\pi / 2)+\arcsin (t / 2))=-t / 2
$$


Now we shall apply the cosine law for $\Delta_{A B B^{\prime}}$.

$$
b^{2}=a^{2}+d^{2}-2 a d \cos (\phi) \leq a^{2}+d^{2}+a d t .
$$

Since $B A$ is a chord of the circle with centre $M$ and radius $R$ having central angle $\beta^{\prime}$, we have that $a=|B A|<\overparen{B A}=R \beta^{\prime}$. Using this and (31), we get the estimates

$$
b^{2}<\left(R \beta^{\prime}\right)^{2}+\frac{4\left(\varepsilon \beta^{\prime}\right)^{2}}{4-t^{2}}+\frac{2 R t \varepsilon\left(\beta^{\prime}\right)^{2}}{\sqrt{4-t^{2}}}
$$

and

$$
\frac{b^{2}}{\beta^{\prime 2}}<R^{2}+\frac{4 \varepsilon^{2}}{4-t^{2}}+\frac{2 R t \varepsilon}{\sqrt{4-t^{2}}}=\left(R+\frac{t \varepsilon}{\sqrt{4-t^{2}}}\right)^{2}+\varepsilon^{2} .
$$

Now we are ready to estimate $b / \beta^{\prime}$.

$$
\frac{b}{\beta^{\prime}}-\left(R+\frac{t \varepsilon}{\sqrt{4-t^{2}}}\right)=\frac{\left(b / \beta^{\prime}\right)^{2}-\left(R+\frac{t \varepsilon}{\sqrt{4-t^{2}}}\right)^{2}}{\left(b / \beta^{\prime}\right)+R+\frac{t \varepsilon}{\sqrt{4-t^{2}}}}<\frac{\varepsilon^{2}}{1}=\varepsilon^{2},
$$

where we used that $R \geq 1$. Thus

$$
\frac{b}{\beta^{\prime}}<R+\frac{t \varepsilon}{\sqrt{4-t^{2}}}+\varepsilon^{2} .
$$

Now we estimate the quotient $\frac{\beta}{\beta^{\prime}}$ using (28), (29) and (32).

$$
\begin{aligned}
\frac{\beta}{\beta^{\prime}} & <\frac{b}{\beta^{\prime}} \frac{1}{s}\left(1+\varepsilon^{2}\right)<\frac{R+\frac{t \varepsilon}{\sqrt{4-t^{2}}}+\varepsilon^{2}}{R+(\varepsilon / 2) \cdot \sqrt{4-R^{2}} \varepsilon-8 \varepsilon^{2}}\left(1+\varepsilon^{2}\right)= \\
& =\left(1-\frac{(\varepsilon / 2) \cdot \sqrt{4-R^{2}}-\frac{t \varepsilon}{\sqrt{4-t^{2}}}-9 \varepsilon^{2}}{R+(\varepsilon / 2) \cdot \sqrt{4-R^{2}}-8 \varepsilon^{2}}\right)\left(1+\varepsilon^{2}\right) .
\end{aligned}
$$

If we show that

$$
\frac{(\varepsilon / 2) \cdot \sqrt{4-R^{2}}-\frac{t \varepsilon}{\sqrt{4-t^{2}}}-9 \varepsilon^{2}}{R+(\varepsilon / 2) \cdot \sqrt{4-R^{2}}-8 \varepsilon^{2}} \geq \varepsilon^{2},
$$

then we are done since then

$$
\frac{\beta}{\beta^{\prime}}<\left(1-\varepsilon^{2}\right)\left(1+\varepsilon^{2}\right)=1-\varepsilon^{4}=q<1 .
$$


Since $R+(\varepsilon / 2) \cdot \sqrt{4-R^{2}}-8 \varepsilon^{2} \leq 2+1=3$, it is enough to show that

$$
(\varepsilon / 2) \cdot \sqrt{4-R^{2}}-\frac{t \varepsilon}{\sqrt{4-t^{2}}}-9 \varepsilon^{2} \geq 3 \varepsilon^{2} .
$$

In turn, by $\sqrt{4-R^{2}} \geq \sqrt{4-t^{2}}$, it is enough to show that

$$
(\varepsilon / 2) \cdot \sqrt{4-t^{2}}-\frac{t \varepsilon}{\sqrt{4-t^{2}}}-9 \varepsilon^{2} \geq 3 \varepsilon^{2},
$$

which is equivalent to

$$
\frac{4-t^{2}-2 t}{\sqrt{4-t^{2}}} \geq 24 \varepsilon
$$

Since $R=1.227$, we have $t<R+\varepsilon<1.227+10^{-6}<1.228$. Then $t<1.23<$ $\sqrt{5}-1$, and thus $4-t^{2}-2 t>0$. Therefore, $(33)$ is equivalent to

$$
\left(4-t^{2}-2 t\right)^{2} \geq\left(4-t^{2}\right)(24 \varepsilon)^{2}
$$

In order to check this inequality, it is enough to show that

$$
t^{4}+4 t^{3}-4 t^{2}-16 t+15.999 \geq 0
$$

considering that that $\varepsilon<10^{-6}$. One can check that if $0<t \leq 1.228$, then (34) is indeed satisfied, and we are done. 


\section{References}

[1] A. S. Besicovitch, On Kakeya's problem and a similar one, Math. Z. 27 (1928), 312-320.

[2] F. Cunningham, Jr., The Kakeya problem for simply connected and for star-shaped sets, Amer. Math. Monthly, 78 (1971), 114-129.

[3] F. Cunningham, Jr., Three Kakeya problems. Amer. Math. Monthly, 81 (1974), 582-592.

[4] M. Csörnyei, K. Héra and M. Laczkovich, Closed sets with the Kakeya property, Mathematika 63, (2017), 184-195.

Department of Analysis, Institute of Mathematics, Eötvös Loránd University

Budapest, Pázmány Péter sétány 1/C, 1117 Hungary

e-mail: herakornelia@gmail.com (Kornélia Héra)

laczk@cs.elte.hu (Miklós Laczkovich) 